\title{
Nonlinear Optimal Control Techniques for Vibration Attenuation Using Magnetostrictive Actuators
}

\author{
William S. Oates ${ }^{1}$ and Ralph C. Smith ${ }^{2}$ \\ Center for Research in Scientific Computation \\ Department of Mathematics \\ North Carolina State University \\ Raleigh, NC 27695
}

\begin{abstract}
This paper addresses the development of a nonlinear control design for attenuating structural vibrations using magnetostrictive transducers operating in nonlinear and highly hysteretic operating regimes. We consider as a prototype a thin plate subjected to exogenous pressure waves and controlled via Terfenol-D transducers at the plate edges; however the methodology is sufficiently general to encompass a wide range of structures and magnetic transducer designs. Hysteresis inherent to the transducer materials is quantified using a homogenized energy framework and the resulting nonlinear constitutive relations are used to construct a PDE representation and corresponding finite dimensional model of the structural system. We employ optimal control theory to construct nonlinear open loop control inputs which accommodate the hysteresis inherent to the transducers but are not robust with regard to unmodeled dynamics or disturbances. Robustness is incorporated by employing perturbation techniques to provide linear feedback laws acting on measured disturbances. As illustrated via numerical examples, the resulting hybrid control design provides excellent control authority and robustness for transducers operating in hysteretic and nonlinear regimes.
\end{abstract}

Keywords: Nonlinear optimal control, perturbation control, hysteresis, nonlinear magnetic transducers

\section{Introduction}

The active and passive attenuation of vibrations in aeronautic, aerospace, automotive, and industrial systems constitutes a fundamental component of structural and structural acoustic design. These vibrations can be due to a wide range of exogenous inputs including adjacent machinery, environmental inputs, or impinging acoustic or fluid fields, and can be periodic, quasiperiodic, or random in nature. For certain systems, passive damping

\footnotetext{
${ }^{1}$ Email: wsoates@ncsu.edu, Telephone: (919) 515-2386

${ }^{2}$ Email: rsmith@eos.ncsu.edu, Telephone: (919) 515-7552
} 
techniques prove adequate and are advantageous due to their simplicity and robustness. However, passive vibration control often comes at the cost of added weight and size, and this approach often proves inadequate in moderate to high frequency regimes. For such applications, active or semi-active vibration control is required. Consideration of active designs is further motivated by increased development and utilization of highly flexible and lightweight structural components having little inherent damping - e.g., composites and polymers - and increasingly stringent design criteria and operating regimes.

Over the past two decades, a range of smart materials - including piezoelectric compounds, relaxor ferroelectric materials, magnetostrictive compounds, and shape memory alloys (SMA) - have emerged as viable alternatives for both passive and active vibration control. Their advantages arise from number of factors including multifunctionality (e.g., both actuator and sensor capabilities), large force or strain generation, high frequency and broadband actuator capabilities (excluding SMA), and the potential for minimal weight increase. At low drive levels, the dynamics of ferroelectric and ferromagnetic actuators can often be adequately approximated using linear constitutive relations which subsequently form the basis for linear structural models and control designs. In moderate and high drive regimes, however, inherent hysteresis and constitutive nonlinearities sufficiently dominate material behavior to require inclusion in models and model-based control designs.

As detailed in [40], there exists a wide range of techniques for modeling the hysteresis inherent to ferroelectric (e.g., PZT, PMN below the glass transition temperature), ferromagnetic (e.g., Terfenol-D, steel) and ferroelastic (e.g., SMA) compounds. Three modeling classes which provide unified characterization frameworks for ferroelectric, ferromagnetic, and ferroelastic - collectively termed ferroic - compounds are domain wall models [25, 28, 42], Preisach models [22, 35, 37], and homogenized energy models [38, 41, 44, 45]. The domain wall models are efficient to implement but require a priori knowledge of turning points to guarantee closure of biased minor loops. For feedback design, this can prove problematic since turning points are dictated by state measurements or estimates for the underlying structure. For certain operating regimes, the utility of a model-based optimal control design utilizing a domain wall model is illustrated in [39] — for general tracking and regulation application however, feedback control laws exploiting domain wall models should be used with care due to the inherent nonclosure property. Preisach models were originally developed for magnetic materials $[17,35]$ and have been exploited for model-based control design for all three classes of compounds. The primary advantage of this technique is also one of its primary limitations, namely its general mathematical nature. For systems in which the physics is poorly understood or difficult to quantify, this generality is a definite advantage. However, it is difficult to correlate parameters in the framework with measured quantities, and extensions to the theory are required to accommodate noncongruency, reversibility, nonclosure due to relaxation mechanisms, and stress or temperature dependencies $[15,16,40]$. In this paper, we employ the homogenized energy framework due to 
its energy basis and flexibility with regard to numerous operating conditions. Details regarding this framework, including its relation to certain extended Preisach formulations, can be found in [40].

The majority of control designs utilizing smart material transducers have focused on linear models which are effective in low to moderate drive level regimes [4, 6, 32, 33, 34]. Within the context of nonlinear hysteresis models, there are two primary approaches: (i) linear control design utilizing model inverses employed as inverse filters to approximately linearize the transducer response, and (ii) nonlinear control design. An overview of the former approach using a piecewise linear Preisach representation can be found in [46] and the use of this technique for robust control design utilizing the homogenized energy framework is detailed in [29]. This technique has the advantage that it permits linear adaptive, optimal, classical or robust control design. The primary disadvantage resides in the fact that inputs to the model inverse often are more difficult to interpret physically than direct inputs to the transducer. In category (ii), nonlinear control theory is used to ascertain inputs to the actuator which yield the desired system response - e.g., effective vibration attenuation or high accuracy tracking. This category includes the domain wall-based optimal design in [39] as well as nonlinear designs of the type detailed in $[21,3,23]$. Schematics illustrating the two approaches are provided in Figure 1.

In this paper, we develop a nonlinear optimal control technique for magnetostrictive transducers which utilizes the homogenized energy framework to quantify the hysteretic map between input magnetic fields $H$
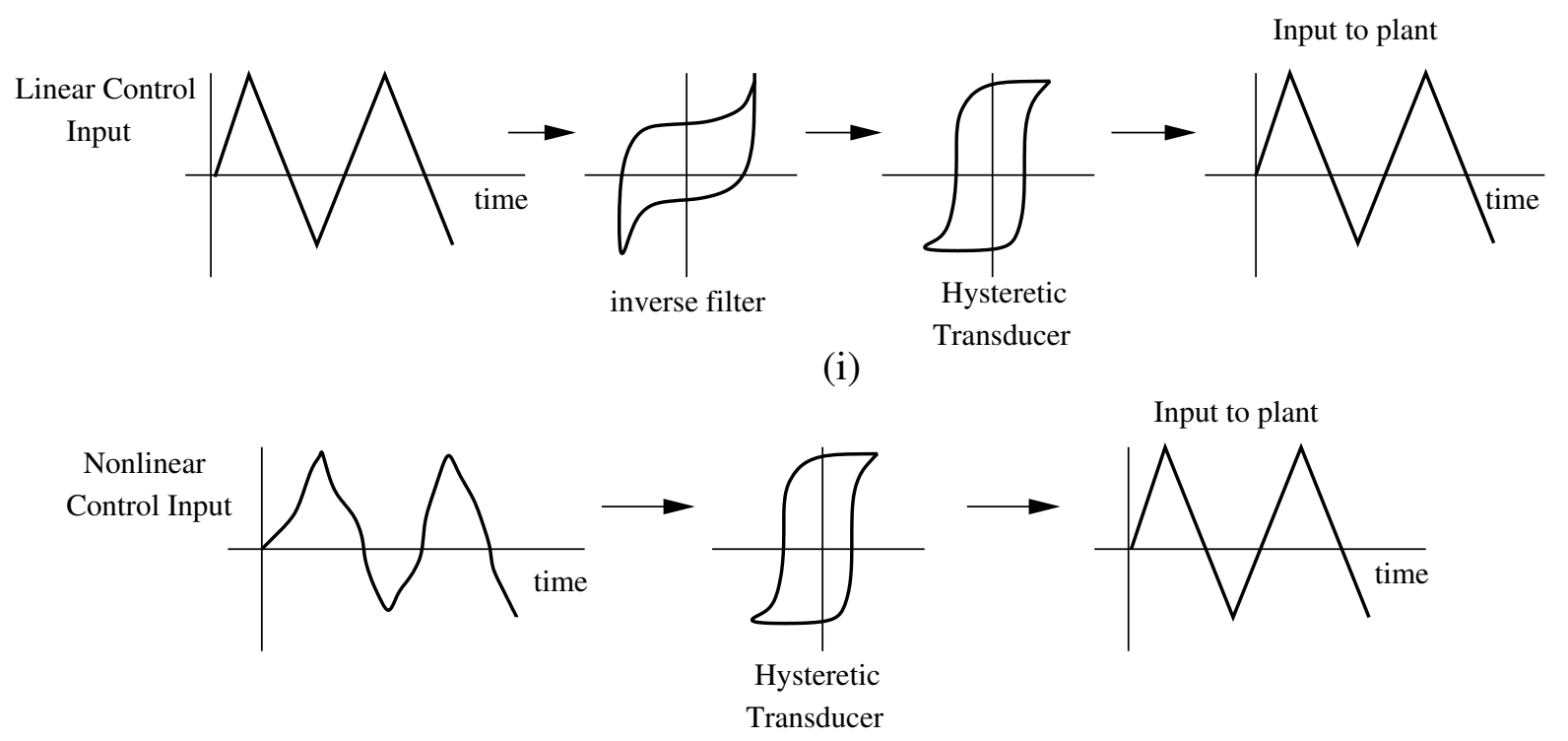

(ii)

Figure 1: (i) Linear control design employing an inverse filter, and (ii) nonlinear control design. 
and the magnetization $M$ and quadratic magnetization - strain $(M-\varepsilon)$ relation. Magnetostrictive actuators have been considered for a number of vibration attenuation applications including the reduction of chatter in machining operations, vibration attenuation in transmissions and axle differentials, and damping of large flexible structures $[12,13,36]$. To provide a prototype for which the structural physics is well-understood but finitedimensional models are relatively high-order, we consider the control of vibrations in a cantilever plate using edge-mounted Terfenol-D transducers as depicted in Figure 2. This simplified construct permits us to focus the discussion on attributes of the nonlinear control design for a 2-D structure without the more complicated system models required for the previously mentioned applications. In this context, we illustrate the effectiveness of the technique for systems having over 150 degrees of freedom which provides an indication that the control method will be feasible when applied to finite element models for more complex applications and designs.

The hybrid nonlinear control design is comprised of an open loop component, derived from nonlinear, finitedimensional control theory, and a feedback component obtained through perturbation techniques. The open loop control accommodates the hysteresis and constitutive nonlinearities inherent to magnetostrictive transducers but is not robust with regard to unmodeled dynamics or uncertainties in operating conditions. Robustness is obtained by linearizing the system about the optimal trajectory and control, and feeding back on perturbations from the open loop system - e.g., see [5] for analysis of perturbation control for general nonlinear systems or [27] for certain algorithms. This approach has the advantage that the resulting perturbation system is linear with quadratic constraints and hence LQR theory can be applied to facilitate implementation. The implementation procedure can be summarized as follows. (i) The nonlinear two-point boundary value problem required to obtain an open loop control is solved off-line and the resulting optimality system is stored. (ii) During numerical or experimental implementation of the method, perturbations from the optimality system are measured and feedback via either LQR or classic methods to compensate for unmodeled dynamics or uncertainties in operating conditions. As demonstrated through numerical examples, the resulting hybrid control method is robust and efficient to implement. Hence it presents an efficient alternative to dynamic programming solutions [5] or state-dependent Ricatti solutions [1] for real-time implementation of nonlinear control laws.

The results presented here differ from those of [39] in two fundamental aspects: (i) the homogenized energy framework provides significantly more flexibility for control design using a range of smart material actuators than the domain wall model employed in [39], and (ii) the development and illustration of the theory in the context of a 2-D plate model rather than the 1-D beam model employed in [39] demonstrates the potential for implementing the method with larger scale FE models used to model the dynamics of more general vibration attenuation systems.

The development of the optimal control method is presented as follows. Section 2 summarizes magne- 
tostrictive material behavior and the homogenized energy model. The magnetically actuated plate structure is developed in Section 3. In Section 4, the optimal control problem is discussed. Linear optimal control results illustrate the need for nonlinear control when field levels reach moderate to high regimes. The nonlinear optimal control method is then developed and validated numerically for the cases of free and forced vibration. Finally perturbation control is implemented to improve robustness to operating uncertainties.

\section{Material Behavior and Hysteresis Model}

Magnetostrictive materials are a special class of magnetic compounds that undergo a shape change when exposed to a magnetic field. The deformation is a manifestation of local magnetic moments that align with the applied field. Under small fields, regions of like magnetization called domains move in an approximately reversible manner - creating small strains and minimal hysteresis. As fields increase, irreversible domain wall motion and rotation of domains develop creating significant hysteresis and nonlinearity.

The pseudobinary compound Terfenol-D $\left(\mathrm{Tb}_{0.3} \mathrm{Dy}_{0.7} \mathrm{Fe}_{1.9}\right)$ is increasingly employed in actuator design due to its large force and strain response under a magnetic field [6, 9]. Magnetostriction in the approximately linear range is on the order of 500 microstrain, whereas strain in excess of 1000 microstrain can be achieved in the nonlinear range. Certain transducer designs can generate forces up to $550 \mathrm{~N}$ with broadband capability from DC up to $20 \mathrm{kHz}[20]$.

The magnetostrictive transducer employed in the structural control problem is described in detail by Dapino et al. [13]. As shown in Figure 2(a), the actuator primarily consists of a Terfenol-D rod, a surrounding wire solenoid, a permanent magnet, and a spring washer/compression bolt assembly to prestress the rod. A current is applied to the solenoid which generates a magnetic field. The magnetic field produces magnetic flux, magnetization, and strain in the Terfenol-D rod. The rod is prestressed to reduce tensile loads during operation and to increase actuator displacement [18]. The permanent magnet serves to bias the magnetization on the Terfenol-D rod such that bi-directional strains are produced by a time-varying magnetic field with zero bias and to provide uniform flux patterns [11]. Bi-directional strain can also be achieved by biasing the magnetic field, but this results in substantial power losses from ohmic heating which necessitates use of liquid-cooled transducers. Trade-offs between size and weight of permanent magnets and power loss from ohmic heating must be considered for a given application.

\subsection{Homogenized Energy Model}

A one-dimensional magnetostrictive constitutive law is used to model the Terfenol transducers. The homogenized energy model focuses on the nonlinear, hysteretic $H-M$ behavior while assuming linear stress-strain 

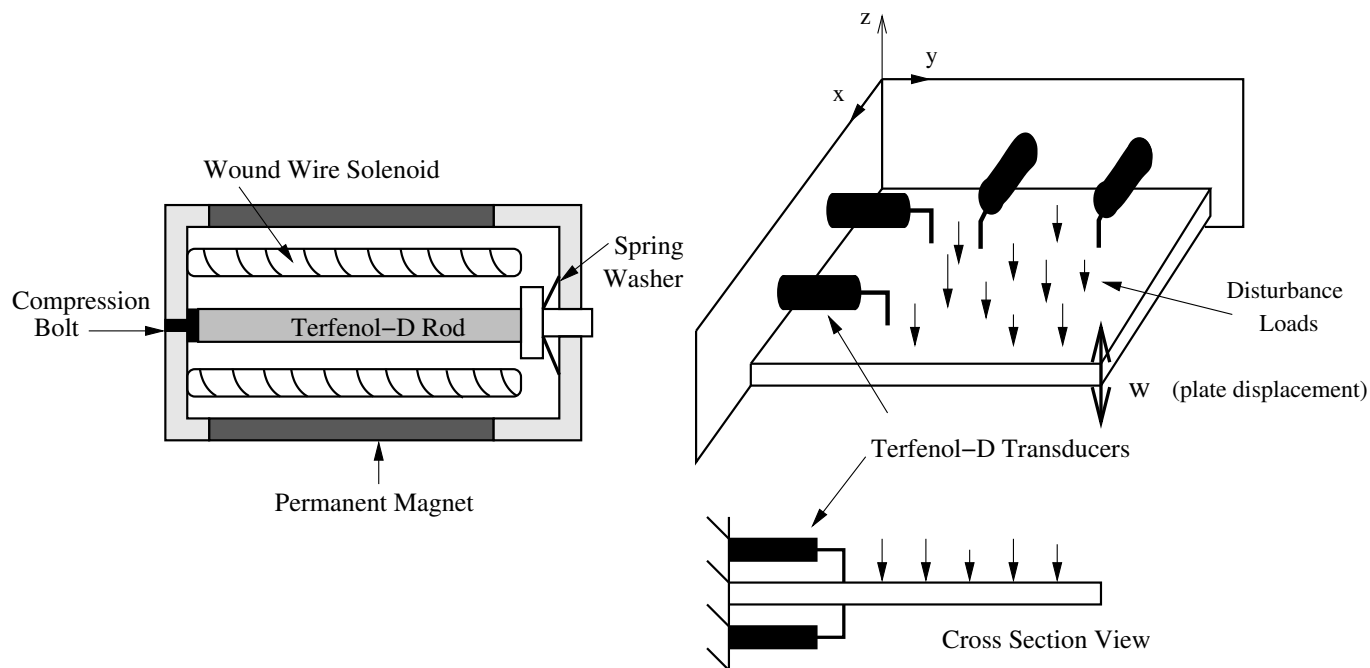

(a)

(b)

Figure 2: (a) Terfenol-D transducer design, and (b) transducer configuration for attenuating transverse plate vibrations.

behavior. A brief summary detailing the constitutive model is presented here. Details regarding the model development are found in [40,41].

To summarize, a Gibbs relation at the mesoscopic length scale is used to quantify the local magnetization $\bar{M}$ as a function of the applied field $H$ for regimes in which relaxation mechanisms may be negligible or significant. The model is extended to macroscopic scales through stochastic homogenization techniques. The Gibbs relation is

$$
G(M)=\Psi(M)-\mu_{0} H M
$$

where $\Psi(M)$ is the Helmholtz energy [40] and $\mu_{0}$ is the permeability of free space.

As detailed in Chapter 7 of [40], the constitutive relations are

$$
\begin{aligned}
& \sigma(t)=E^{M} \varepsilon(t)-a_{1}\left([M(H)](t)-M_{0}\right)^{2} \\
& {[M(H)](t)=\int_{0}^{\infty} \int_{-\infty}^{\infty} \nu_{1}\left(H_{c}\right) \nu_{2}\left(H_{I}\right)\left[\bar{M}\left(H+H_{I} ; H_{c}, \xi\right)\right](t) d H_{I} d H_{c}}
\end{aligned}
$$

where $\sigma(t)$ is the uniaxial stress, $\varepsilon(t)$ is the elastic strain, $E^{M}$ is the elastic modulus at constant magnetization, $a_{1}$ is the magnetostrictive coefficient, $M$ is the magnetization and $M_{0}$ includes magnetization from the permanent magnet and the initial internal state of the material. Here, $H_{I}$ is the interaction field, $H_{c}$ is the coercive field 
and $\xi$ denotes the initial distribution of magnetic variants. The stochastic homogenization technique used to formulate the macroscopic magnetization $M$ in terms of local values $\bar{M}$ is based on the assumption that the local coercive field $H_{c}$ and interaction field $H_{I}$ are manifestations of underlying distributions rather than constants to accommodate a myriad of local material inhomogeneities such as intergranular residual stress, impurities, and certain anisotropies. The corresponding densities are designated by $\nu_{1}\left(H_{c}\right)$ and $\nu_{2}\left(H_{I}\right)$. As detailed in [41], one choice for these densities is

$$
\nu_{1}\left(H_{c}\right) \nu_{2}\left(H_{I}\right)=c_{1} c_{2} e^{-\left[\ln \left(H_{c} / \bar{H}_{c}\right) / 2 c\right]^{2}} e^{-H_{I}^{2} / 2 b^{2}}
$$

where $\bar{H}_{c}$ is the average coercive field, $c$ quantifies the coercive field variability, $b$ is the variance of the interaction field, and $c_{1}$ and $c_{2}$ are scaling parameters. The proposed densities are implemented to reduce parameter estimation. Model comparison to experimental results can be found in [41].

For many operating regimes, thermal activation mechanisms such as magnetic after-effects and accommodation [24] significantly affect the constitutive behavior. These effects are incorporated in the framework through Boltzmann's relations

$$
\mu(G)=C e^{-G V / k T}
$$

which balances the Gibbs and relative thermal energies. Here $G$ is the Gibbs energy, $V$ is a representative volume element, $k$ is Bolztmann's constant, and $T$ is temperature. The constant $C$ is specified to ensure integration to unity.

In this case, the local magnetization is defined by

$$
\bar{M}=x_{+}\left\langle M_{+}\right\rangle+x_{-}\left\langle M_{-}\right\rangle
$$

where $x_{+}$and $x_{-}$respectively denote the volume fraction of moments having positive and negative orientations. The variables $\left\langle M_{+}\right\rangle$and $\left\langle M_{-}\right\rangle$are the average magnetizations corresponding to the volume fractions of moments. The differiential equations governing evolution of $x_{+}$and $x_{-}$is based on material-dependent parameters that define the likelihoods that moments switch from positive to negative, and conversely. These likelihoods account for the observed thermal relaxation mechanisms. Details describing the governing equations are given in [41].

In certain operating regimes, thermal relaxation is negligible and in which case the relation (5) limits to the piecewise linear relation

$$
\bar{M}\left(H+H_{I} ; H_{c}, \xi\right)=\chi_{m}\left(H+H_{I}\right)+M_{R} \delta\left(H+H_{I} ; H_{c}, \xi\right)
$$


where $M_{R}$ is the magnitude of the local magnetization variant. The variable $\delta$ is equal to one if the magnetization variant is in the positive direction and negative one if oriented in the negative direction. The magnetic susceptibility is defined by $\chi_{m}$.

The local magnetization $M_{R}$ given in (6) will switch when magnetic variants diametrically opposed to the effective field $\left(H_{e}=H+H_{I}\right)$ reach the coercive field. The switching behavior is modeled by introducing a semi-infinite set of magnetic variants that correspond to the distribution of effective fields and coercive fields. Details regarding the numerical implementation of the switching behavior and the numerical integration of $(2)$ are provided in $[40,45]$. The constitutive response predicted by the homogenized free energy model is illustrated in Figure 3. The local magnetization given by (6) has been used. Although this is a linear piecewise function, the typical nonlinearities associated with magnetostrictive materials are captured by the local relation for $\bar{M}$.

For purposes of developing the structural model and control design, the local magnetization given by (6) is implemented where thermal activation mechanisms are neglected.

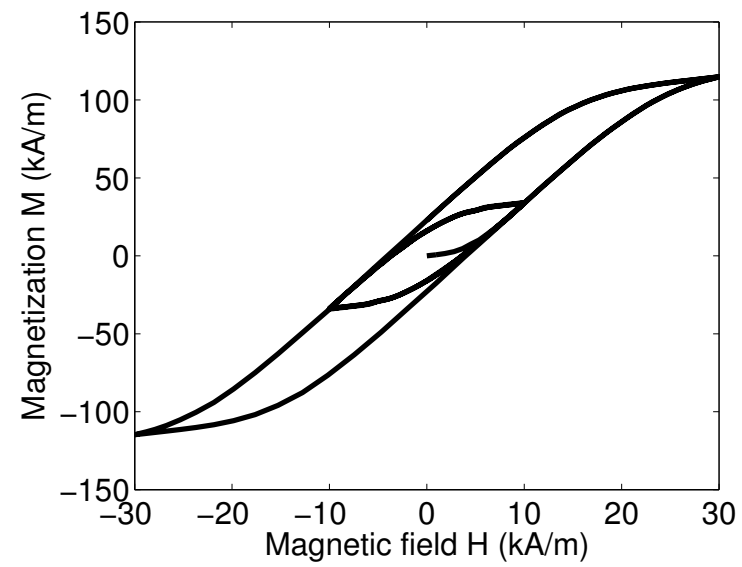

(a)

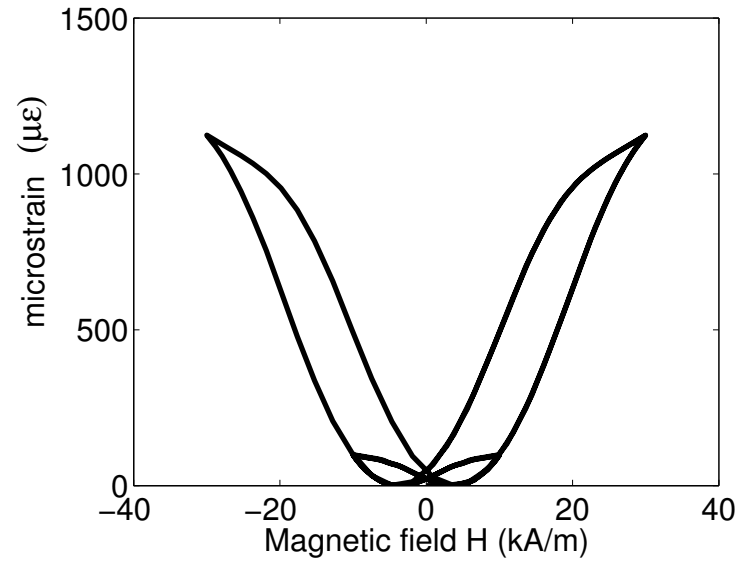

(b)

Figure 3: (a) Macroscopic magnetization $M$ versus magnetic field $H$ computed using (2), and (b) microstrain $\mu \varepsilon$ versus magnetic field $H$ computed from (2) under zero external stress. 


\section{Structural Model}

The structure under consideration consists of Terfenol transducers positioned along two fixed edges of a thin plate as depicted in Figure 2. Eight transducers are oriented in four pairs with each pair attached to the plate by a moment arm that is perpendicular to the plate. Diametrically out-of-phase currents applied to each actuator couple generate a moment on the plate.

\subsection{Plate With Nonlinear Actuators}

The thin plate structure is modeled using classical plate theory [47]. The equation of motion describing transverse plate displacement $w$ is decoupled from in-plane displacements by focusing on small strain induced by plate vibration. Since the control problem is concerned with transverse plate vibration, we focus only on the dynamic equation for $w$.

The balance of forces and moments for the plate result in the strong form of the equation of motion,

$$
\rho_{p} \frac{\partial^{2} w}{\partial t^{2}}-\frac{\partial^{2} \mathcal{M}_{x}^{\text {int }}}{\partial x^{2}}-2 \frac{\partial^{2} \mathcal{M}_{x y}^{\text {int }}}{\partial x \partial y}-\frac{\partial^{2} \mathcal{M}_{y}^{\text {int }}}{\partial y^{2}}=\frac{\partial^{2} \mathcal{M}_{x}^{\text {mag }}}{\partial x^{2}}+\frac{\partial^{2} \mathcal{M}_{y}^{\text {mag }}}{\partial y^{2}}+g
$$

where $\rho_{p}$ denotes the density of the plate, $\mathcal{M}_{x}^{\text {int }}$ and $\mathcal{M}_{y}^{\text {int }}$ include the internal elastic and damping moment components in the $x$ and $y$ directions, and $\mathcal{M}_{x y}^{i n t}$ is the in-plane twisting moment. The external moments generated by the Terfenol transducers are represented by $\mathcal{M}_{x}^{\text {mag }}$ and $\mathcal{M}_{y}^{\text {mag }}$ in the $x$ and $y$ directions, respectively. No twisting moments are applied by the transducers, so the component $\mathcal{M}_{x y}^{\text {mag }}$ is zero. The external disturbance load distributed along the plate surface is denoted by $g$. A detailed description of the equations describing the moment-displacement relations are given in the Appendix. Spatial variations in density, moment of inertia and compliance near the Terfenol transducers and connecting moment arms are assumed to be negligible.

The plate geometry is represented by the length $\ell_{x}$, width $\ell_{y}$ and thickness $h$. Values used in the model are given in Table 1 . The plate is assumed to be clamped along the fixed edges $[x, 0]$ and $[0, y]$ and free along $\left[\ell_{x}, y\right]$ and $\left[x, \ell_{y}\right]$ as shown in Figure 2(b). The cross-sectional area of the Terfenol rod is denoted by $A_{m a g}$ and we let $\ell_{r}$ deonote the length of the moment arm from the actuator to the surface of the plate. The location of the four connecting rods are designated by $\Omega_{i}, i=1, \ldots, 4$. Let $H_{1(i)}$ and $H_{2(i)}, i=1, \ldots, 4$ respectively denote the field inputs to the top and bottom actuators in each pair and let $\mathbf{H}(t)=\left[H_{1(1)}(t), H_{2(1)}(t), \ldots, H_{1(4)}(t), H_{2(4)}(t)\right]$ denote the complete set of inputs. The external moments generated by the Terfenol actuators can then be expressed as 


$$
\begin{aligned}
& \mathcal{M}_{x}^{m a g}(\mathbf{H}(t), x, y)=-\mathcal{K}^{M} \sum_{i=1}^{4}\left[M_{1(i)}^{2}\left(H_{(i)}(t)\right)-M_{2(i)}^{2}\left(H_{(i)}(t)\right)\right] \chi_{x(i)}(x, y) \\
& \mathcal{M}_{y}^{m a g}(\mathbf{H}(t), x, y)=-\mathcal{K}^{M} \sum_{i=1}^{4}\left[M_{1(i)}^{2}\left(H_{(i)}(t)\right)-M_{2(i)}^{2}\left(H_{(i)}(t)\right)\right] \chi_{y(i)}(x, y)
\end{aligned}
$$

where $\mathcal{K}^{M}=a_{1} A_{\text {mag }}\left(h / 2+\ell_{r}\right)$ and $M_{1(i)}(t)$ and $M_{2(i)}(t)$ are the magnetizations modeled by $(2)$. The characteristic functions

$$
\begin{aligned}
& \chi_{x(i)}(x, y)= \begin{cases}1 & ,(x, y) \in \Omega_{i}, i=1,2 \\
0 & , \text { otherwise }\end{cases} \\
& \chi_{y(i)}(x, y)= \begin{cases}1 & ,(x, y) \in \Omega_{i}, i=3,4 \\
0 & , \text { otherwise }\end{cases}
\end{aligned}
$$

designate that actuators 1 and 2 along $[0, y]$ generate moments in the $x$-direction whereas actuators 3 and 4 along $[x, 0]$ generate moments in the $y$-direction.

The control design is simplified by reducing the number of control inputs from eight to four. The time varying control inputs are computed for each of the four top transducers and the control inputs on the bottom four actuators are defined by $H_{2(i)}(t)=-H_{1(i)}(t)$. The magnetization is then defined by the relation $M_{1(i)}=$ $\widetilde{M}_{(i)}+M_{0}$ and $M_{2(i)}=-\widetilde{M}_{(i)}+M_{0}$ which assumes negligible differences between the magnetization driven by the fields applied on the top and bottom actuators. This yields the relation

$$
\begin{aligned}
& \mathcal{M}_{x}^{\text {mag }}(\mathbf{H}(t), x, y) \cong \sum_{i=1}^{4} \mathcal{M}_{(i)}\left(H_{(i)}(t)\right) \chi_{x(i)}(x, y) \\
& \mathcal{M}_{y}^{\text {mag }}(\mathbf{H}(t), x, y) \cong \sum_{i=1}^{4} \mathcal{M}_{(i)}\left(H_{(i)}(t)\right) \chi_{y(i)}(x, y)
\end{aligned}
$$

where

$$
\mathcal{M}_{(i)}\left(H_{(i)}(t)\right)=-\mathcal{K}^{M}\left[4 M_{0} \widetilde{M}_{(i)}\left(H_{(i)}(t)\right)\right]
$$

which is an approximation of actual magnetostrictive material behavior when hysteresis and nonlinearities are present. The control law can be improved by including all eight transducers as individual inputs, but for the purposes of demonstrating the control design the simpler four input model is used.

As detailed in [40], the strong model formulation (7) can be rewritten in the weak form 


$$
\int_{\Omega}\left(\rho \frac{\partial^{2} w}{\partial t^{2}} \Phi-\mathcal{M}_{x}^{i n t} \frac{\partial^{2} \Phi}{\partial x^{2}}-2 \mathcal{M}_{x y}^{i n t} \frac{\partial^{2} \Phi}{\partial x \partial y}-\mathcal{M}_{y}^{i n t} \frac{\partial^{2} \Phi}{\partial y^{2}}\right) d \omega=\int_{\Omega}\left(\mathcal{M}_{x}^{\text {mag }} \frac{\partial^{2} \Phi}{\partial x^{2}}+\mathcal{M}_{y}^{\text {mag }} \frac{\partial^{2} \Phi}{\partial y^{2}}+g \Phi\right) d \omega
$$

where $\Phi \in H_{0}^{2}(x, y)$ and $\Omega=\left[0, \ell_{x}\right] \times\left[0, \ell_{y}\right]$ denotes the plate region.

\subsection{Approximation Method}

The plate displacements can be approximated using either cubic B-splines or cubic Hermite elements. Cubic B-splines are chosen for the control problem primarily because cubic Hermite elements require solving for approximately twice the number of unknown coefficients which may affect computation speed for real-time control. Details describing the attributes of cubic B-splines as well as comparisons to cubic Hermite elements are given in Chapter 8 of [40].

The cubic B-splines are defined over the plate geometry, $\Omega=\left[0, \ell_{x}\right] \times\left[0, \ell_{y}\right]$. The plate is discretized using the points $x_{m}=m h_{x}$ and $y_{m}=n h_{y}$ with $h_{x}=\frac{\ell_{x}}{N_{x}}, h_{y}=\frac{\ell_{y}}{N_{y}}$ and $m=0, \ldots, N_{x}$ and $n=0, \ldots, N_{y}$. The cubic spline product space is defined by

$$
\Phi(x, y)=\phi(x) \phi(y)
$$

The approximate solution to (15) is subsequently given by

$$
w(t, x, y)=\sum_{k=1}^{N_{w}} w_{k}(t) \Phi_{k}(x, y)
$$

where $N_{w}=\left(N_{x}+1\right)\left(N_{y}+1\right)$ and $w_{k}(t)$ are the coefficients to be determined through solution of the weak model formulation.

Approximation of the infinite dimensional model (15) yields the ODE system

$$
\mathbb{M} \ddot{\mathbf{w}}+\mathbb{C} \dot{\mathbf{w}}+\mathbb{K} \mathbf{w}=\mathbf{b M}(\mathbf{H}(t))+\mathbf{g}
$$

where $\mathbb{M}, \mathbb{C}$, and $\mathbb{K}$ are the mass, damping and stiffness matrices. The vector $\mathbf{w}=\left[w_{1}(t), \ldots, w_{N_{w}}(t)\right]^{T}$ represents the coefficients in (17) and $\mathbf{g}$ consolidates disturbance loads. Details describing the components of these matrices and vectors are given in the Appendix and in [40]. As indicated in (54) in the Appendix, the $\left(N_{w}+1\right) \times 4$ matrix $\mathbf{b}$ specifies the spatial components of the inputs. The $4 \times 1$ input vector

$$
\mathbf{M}(\mathbf{H}(t))=\left[\mathcal{M}_{(1)}\left(H_{(1)}(t)\right), \ldots, \mathcal{M}_{(4)}\left(H_{(4)}(t)\right)\right]^{T}
$$


designates the time-dependent influence of the field inputs.

The second-order system can be formulated as a set of first-order equations for implementation in the control design

$$
\begin{aligned}
& \dot{y}(t)=A y(t)+[B(u)](t)+G(t) \\
& y(0)=y_{0}
\end{aligned}
$$

where $y(t)=\left[w_{1}(t), \cdots, w_{N_{w}}(t), \dot{w}_{1}(t), \cdots, \dot{w}_{N_{w}}(t)\right]$. The system matrix and input vector are given by

$$
A=\left[\begin{array}{cc}
0 & \mathbb{I} \\
-\mathbb{M}^{-1} \mathbb{C} & -\mathbb{M}^{-1} \mathbb{K}
\end{array}\right] \quad, \quad B(u)=\left[\begin{array}{c}
0 \\
\mathbb{M}^{-1} \mathbf{b}
\end{array}\right] \mathbf{M}(u)
$$

with a similar definition for the disturbance load $G(t)$. The identity matrix, with dimension $N_{w} \times N_{w}$, is denoted by $\mathbb{I}$. The control input $u$ is the magnetic field $H$ applied to each transducer.

\subsection{Structural Parameters}

Physical parameters employed in the control design are summarized in Table 1. The Terfenol material parameters implemented in the homogenized free energy model are within the range obtained for model fits to an experimental transducer [8]. The plate modulus $E_{p}$, Poisson ratio $\nu_{p}$ and density $\rho_{p}$ are typical for aluminum. The internal damping is assumed to be linear and is represented by $c_{p}$ while viscous air damping is given by $\gamma$. The center location of the four actuator pairs was $(0.105,0.2),(0.105,0.3),(0.15,0.105)$ and $(0.25,0.105)$ in millimeters where the coordinate origin was located at the top left corner of the plate as shown in Figure 2(b). The moment arm is assumed to be rigid with cross-sectional area denoted by $A_{r}$. The cross-section area is used to determine the region over which the moment is applied. Plate dynamics were sufficiently resolved by choosing $N_{x}=N_{y}=4$ in the frequency range considered. The dimension of the state vector $y$ was then $50 \times 1$ due to the inclusion of both displacement and velocity components. When determining the convergence of the numerical approximations, however, we have both simulated and run control problems with state vectors up to $N_{x}=N_{y}=8$ for a total of 162 degrees of freedom. 
Table 1: Parameters for the plate and Terfenol transducer.

\begin{tabular}{l|l}
\hline Plate & Terfenol Transducer \\
\hline$E_{p}=4.1 \times 10^{10} \mathrm{~N} / \mathrm{m}^{2}$ & $E^{M}=7.00 \times 10^{10} \mathrm{~N} / \mathrm{m}^{2}$ \\
$\nu_{p}=0.345$ & $c_{1}=c_{2}=6.1 \times 10^{-5} \mathrm{~m} / \mathrm{A}$ \\
$\rho_{p}=2700 \mathrm{~kg} / \mathrm{m}^{3}$ & $\bar{H}_{c}=3.3 \times 10^{3} \mathrm{~A} / \mathrm{m}$ \\
$c_{p}=2.5 \times 10^{5} \mathrm{Ns} / \mathrm{m}^{2}$ & $c=0.4$ \\
$\gamma=0.18 \mathrm{Ns} / \mathrm{m}^{2}$ & $b=1.5 \times 10^{4} \mathrm{~A} / \mathrm{m}$ \\
$\ell_{x}=0.4 \mathrm{~m}$ & $M_{0}=6.618 \times 10^{4} \mathrm{~A} / \mathrm{m}$ \\
$\ell_{y}=0.6 \mathrm{~m}$ & $a_{1}=0.006 \mathrm{~N} / \mathrm{A}^{2}$ \\
$h=0.0016 \mathrm{~m}$ & $A_{\text {mag }}=0.0064 \mathrm{~m}^{2}$ \\
$\ell_{r}=2.54 \mathrm{~cm}$ & $\chi_{m}=1$ \\
$A_{r}=1 \mathrm{~cm}^{2}$ & \\
\hline
\end{tabular}

\section{Control Design}

The optimal control problem is first summarized to elucidate the technique used in developing the nonlinear control design $[5,26,27,31]$. The following performance index

$$
\bar{J}(u)=\frac{1}{2} y^{T}\left(t_{f}\right) \Pi_{f} y\left(t_{f}\right)+\int_{t_{0}}^{t_{f}}\left[H(y, u, t)-\lambda^{T}(t) \dot{y}\right] d t
$$

is used to develop the optimal control design, where the positive definite matrix $\Pi_{f}$ penalizes large terminal values of the state, $H(y, u, t)$ is the Hamiltonian, and $\lambda(t) \in \mathbb{R}^{2 N_{w}}$ is a set of Lagrange multipliers.

The Hamiltonian is

$$
\begin{aligned}
H(y, \lambda, u, t) & =L(y, u, t)+\lambda^{T}[A y(t)+[B(u)](t)+G(t)] \\
& =\frac{1}{2}\left[y^{T}(t) Q y(t)+u^{T}(t) R u(t)\right]+\lambda^{T}[A y(t)+[B(u)](t)+G(t)]
\end{aligned}
$$

where the Lagrangian $L$ includes the penalties on the states and inputs through the semi-definite maxtrix $Q$ and the positive definite matrix $R$.

The optimal control problem requires solution of the two-point boundary value problem governed by (20) and the adjoint or Lagrange multiplier condition

$$
\begin{aligned}
& \frac{d \lambda}{d t}=-\frac{\partial H}{\partial y} \\
& \lambda\left(t_{f}\right)=\Pi_{f} y\left(t_{f}\right) .
\end{aligned}
$$


The optimal control is determined from the stationary condition,

$$
\frac{\partial H}{\partial u}=0
$$

which results in the nonlinear optimal control input

$$
u^{*}(t)=-R^{-1} \frac{\partial B(u)^{T}}{\partial u} \lambda(t)
$$

\subsection{Control Parameters}

The penalties on the states and inputs are adjusted in the linear and nonlinear control simulations to assess the effect of the hysteretic constitutive behavior of the magnetostrictive transducers on vibration attenuation. Values for each case are given in Table 1. In the linear simulations, two sets of values are used to show control performance when nonlinearity and hysteresis are negligible or significant. The values used in the linear simulations as well as the open loop nonlinear control and perturbation control are given in Table 2. The matrix $Q$ in (23) is given by

$$
Q=\left[\begin{array}{cc}
q_{1} \mathbb{K} & 0 \\
0 & q_{2} \mathbb{M}
\end{array}\right]
$$

The penalties on the inputs are given by $R_{i j}=r_{i} \delta_{i j}$ where $\delta$ is the Kronecker delta symbol with $i, j=1$ to 4 for the inputs on the four Terfenol-D transducer couples. The penalty on the final state is defined by $\Pi_{f}=q_{3} \Pi$ where $\Pi$ is the solution to the algebraic Ricatti equation described in the following section.

Table 2: Parameters used to penalize the states and inputs in the linear and nonlinear control simulations. The penalty

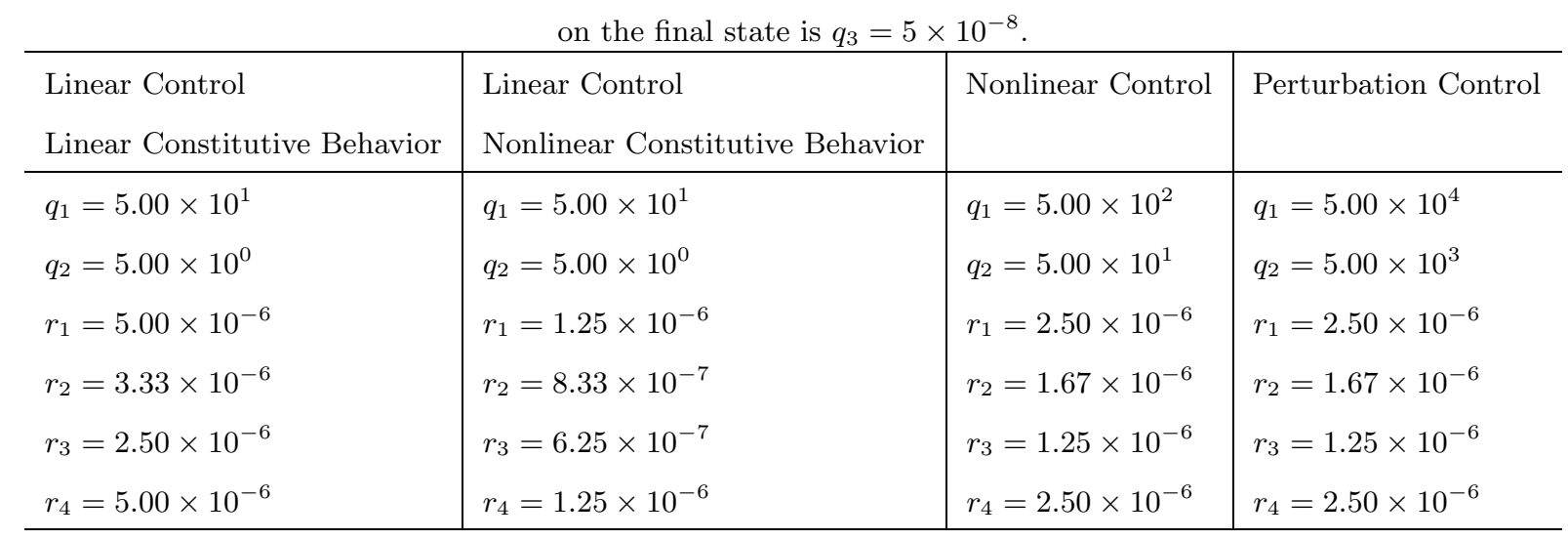




\subsection{Linear Optimal Control}

Linear theory provides acceptable control when input currents to the solenoid are small. It has been experimentally shown that a nearly linear relation exists between magnetic field and strain in the Terfenol transducer when the field is small. In this situation, an approximate model can be attained through linearization about some biased magnetization. In the linear model, the biased magnetization $M_{0}$ includes the fully magnetized internal state of the material and the magnetization from the permanent magnet. This results in a linear input operator where the linear $H-M$ relation $\widetilde{M}_{(i)}(t)=\bar{\chi}_{m} H_{(i)}(t)$ is been substituted into (14) where the macroscopic magnetic susceptibility is $\bar{\chi}_{m}=0.72$. This value is determined numerically from (2) for small field inputs. In this case, the moment from each actuator couple is

$$
\mathcal{M}_{(i)}^{m a g}\left(H_{(i)}(t)\right)=-\mathcal{K}^{M}\left[4 M_{0} \bar{\chi}_{m} H_{(i)}(t)\right]
$$

which is substituted into (12) and (13) to obtain the total applied moment.

Due to the linearity of the constitutive law used here, the input operator is

$$
B=-4 \mathcal{K}^{M} M_{0} \bar{\chi}_{m}\left[\begin{array}{c}
0 \\
\mathbb{M}^{-1} \mathbf{b}
\end{array}\right] \mathbf{H}(t)
$$

where the $4 \times 1$ vector

$$
\mathbf{H}(t)=\left[H_{(1)}(t), \ldots, H_{(4)}(t)\right]^{T}
$$

represents the magnetic field on each actuator couple.

With this approximation, the corresponding first-order system is

$$
\begin{aligned}
& \dot{y}(t)=A y(t)+B u(t)+G(t) \\
& y(0)=y_{0} .
\end{aligned}
$$

The state constraint in (30) and adjoint condition in (24) yields the optimality system

$$
\begin{aligned}
& {\left[\begin{array}{l}
\dot{y}(t) \\
\dot{\lambda}(t)
\end{array}\right]=\left[\begin{array}{cc}
A & -B R^{-1} B^{T} \\
-Q & -A^{T}
\end{array}\right]\left[\begin{array}{l}
y(t) \\
\lambda(t)
\end{array}\right]+\left[\begin{array}{l}
G(t) \\
0
\end{array}\right]} \\
& y\left(t_{0}\right)=y_{0} \\
& \lambda\left(t_{f}\right)=\Pi_{f} y\left(t_{f}\right) .
\end{aligned}
$$


Computing the optimal control requires solution of the two-point boundary value problem defined by the optimality system. In the linear case, a fundamental solution matrix can be obtained by solving the differential Ricatti equation

$$
\begin{aligned}
& -\dot{\Pi}=A^{T} \Pi+\Pi A-\Pi B R^{-1} B^{T} \Pi+Q \\
& \Pi\left(t_{f}\right)=\Pi_{f} .
\end{aligned}
$$

The optimal control input is then defined by,

$$
u^{*}(t)=-R^{-1} B^{T}[\Pi(t) y(t)-r(t)]
$$

where the variable $r(t) \in \mathbb{R}^{2 N_{w}}$ is the solution to the auxiliary differential equation

$$
\begin{aligned}
& \dot{r}(t)=-\left[A-B R^{-1} B^{T} \Pi\right]^{T} r(t)+\Pi G(t) \\
& r\left(t_{f}\right)=0
\end{aligned}
$$

The linear control theory can be further simplified through the assumption that the disturbance force $G$ is periodic on the time interval $\left[t_{0}, t_{f}\right]$. In this case, the appropriate performance index is given by

$$
J(u)=\int_{t_{0}}^{t_{f}} \frac{1}{2}\left[y^{T}(t) Q y(t)+u^{T}(t) R u(t)\right] d t .
$$

This leads to a steady-state controller gain where the fundamental solution matrix is now governed by the algebraic Ricatti equation where $\dot{\Pi}=0$ in (32). The resulting control input is

$$
u^{*}(t)=-R^{-1} B^{T}[\Pi y(t)-r(t)]
$$

Details regarding this approach are given in $[2,14]$.

The control trajectory determined by (36) is used in the following numerical examples to illustrate the need for nonlinear control when the input field reaches a magnitude that induces hysteresis.

\subsubsection{Numerical Example - Truncated External Force}

We first consider the case in which the plate is excited with an external force for a short time interval and then the force is subsequentially set to zero. The disturbance load $G(t)$ was spatially discretized and applied uniformly to the plate with magnitude $25 \mathrm{~N}$ by a summation of five equally weighted frequencies $\left(f^{e}=10,12\right.$, $19,26,37 \mathrm{~Hz}$ ) as given by 


$$
g(t, x, y)= \begin{cases}25 \sum_{i=1}^{5}\left[\sin \left(2 \pi f_{i}^{e} t\right)\right], & t \leq 0.45 \\ 0, & 0.45<t \leq 2.5\end{cases}
$$

where $\sum_{i=1}^{5}\left[\sin \left(2 \pi f_{i}^{e} t\right)\right]=[\sin (2 \pi 10 t)+\cdots+\sin (2 \pi 37 t)]$.

The uncontrolled and controlled displacements at the plate tip $\left[\ell_{x}, \ell_{y}\right]$ are plotted in Figure 4(a). The penalties on the states $Q$ and inputs $R$ are adjusted to ensure the constitutive response computed using (2) is linear. Marginal vibration control is obtained under small input fields. The linear $H-M$ response corresponding to the input control for each actuator pair is illustrated in Figure 4(b).

In Figure 5, the linear control is increased by adjusting $Q$ and $R$ (see Table 2) to illustrate the effect of hysteresis on controlling vibration. The increase in magnetic field creates hysteresis which is not accounted for in the linear control design. When this occurs, the vibration control initially improves, but subsequently degrades due to the phase shift induced by the hysteresis illustrated in Figure 5(b).

\subsubsection{Numerical Example - Periodic External Disturbance Force}

Secondly, we consider the case in which the plate is continuously excited with the external disturbance force (37) (as shown in Figure 2(b)) during the entire simulation. The control is again applied at $t_{0}=0.45 \mathrm{~s}$ and

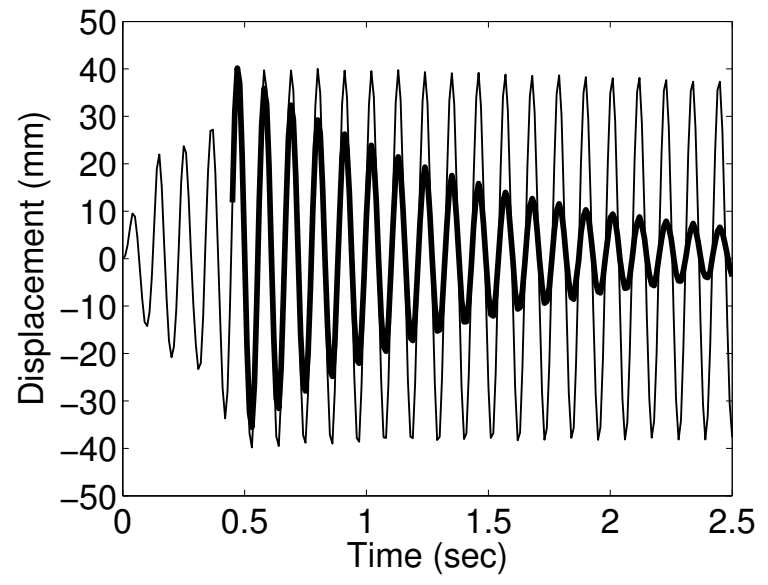

(a)

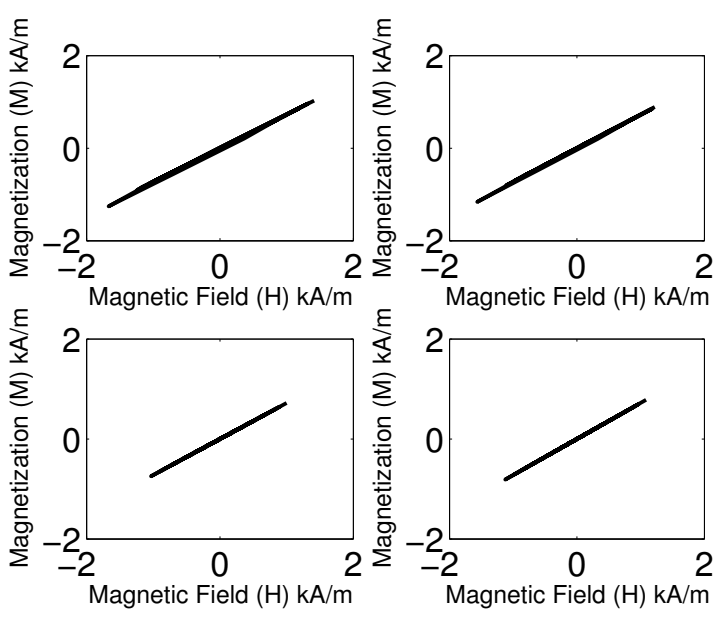

(b)

Figure 4: Performance of the linear feedback control law using the controller gain in (33). The resulting plate displacement for the uncontrolled (-) and controlled response (-) are shown in (a). The relationship between magnetic field and magnetization is shown in (b) for each actuator pair. 


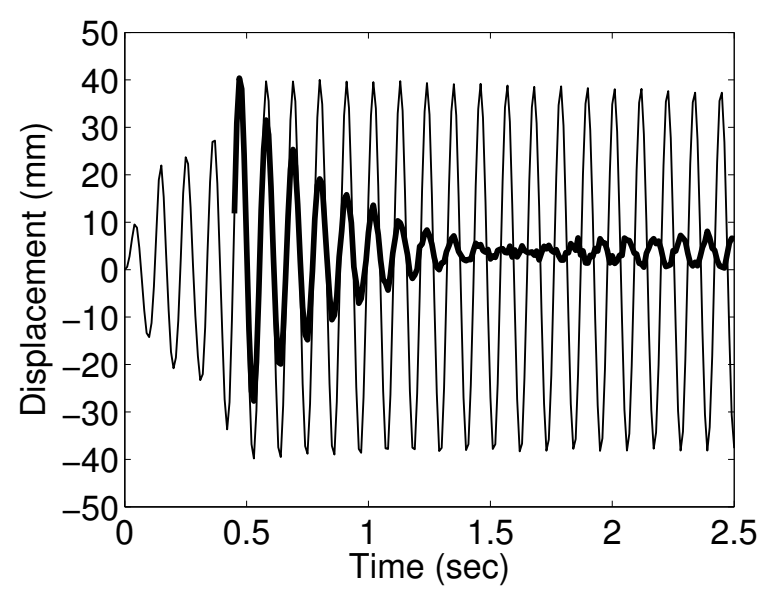

(a)
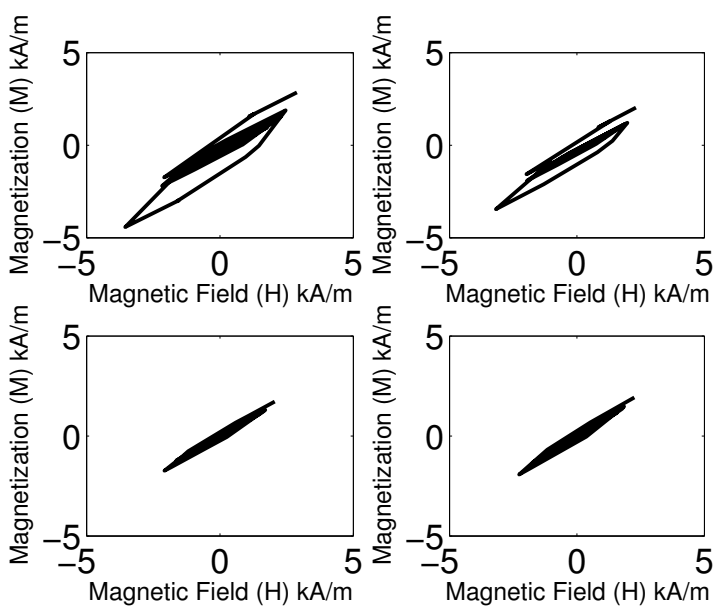

(b)

Figure 5: Linear feedback control law at levels where the transducer model exhibits hysteresis. In this simulation, $Q$ and $R$ were adjusted to increase the input field. The resulting plate displacement for the uncontrolled ( - ) and controlled response (-) are shown in (a) and the corresponding hysteretic $\mathrm{M}-\mathrm{H}$ behavior is shown in (b).

vibration attenuation is marginal as shown in Figure 6. Additionally, the disturbance forces induce nonlinearities in the magnetostrictive actuators from larger field inputs necessary to minimize vibration which is not properly accounted for in the linear control design.

\subsection{Nonlinear Control Method}

The nonlinear control method previously discussed by Smith [39] is applied to the Terfenol plate structure. Key equations describing the numerical technique are summarized to elucidate the approach used. The input operator $B(u)$ contains nonlinearities from the magnetostrictive constitutive law which does not lead to a fundamental matrix solution; therefore an efficient solution in terms of the Riccati equation is not possible. This issue is resolved by solving the optimality system numerically while ensuring the boundary conditions at the initial and final times are satisfied. The first order system of equations is formulated as,

$$
\dot{z}(t)=F(t, z)
$$

where $z=[y, \lambda]^{T}$ and 


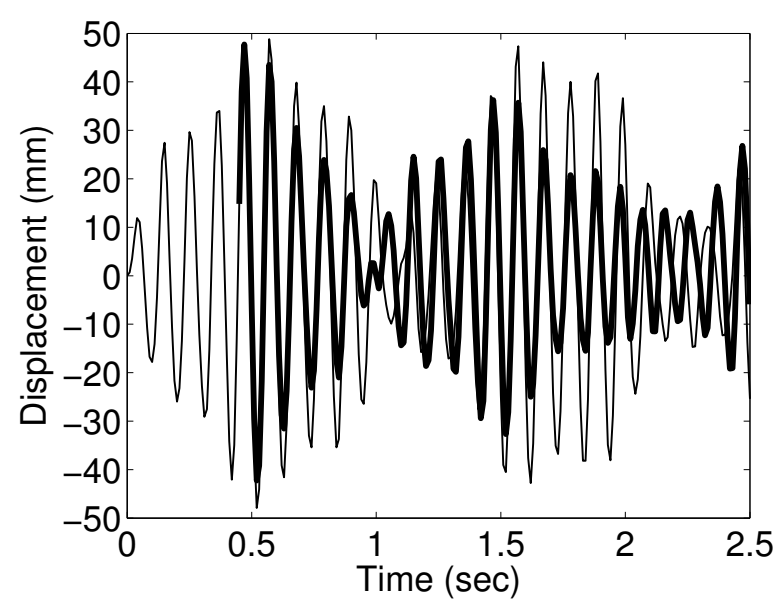

(a)
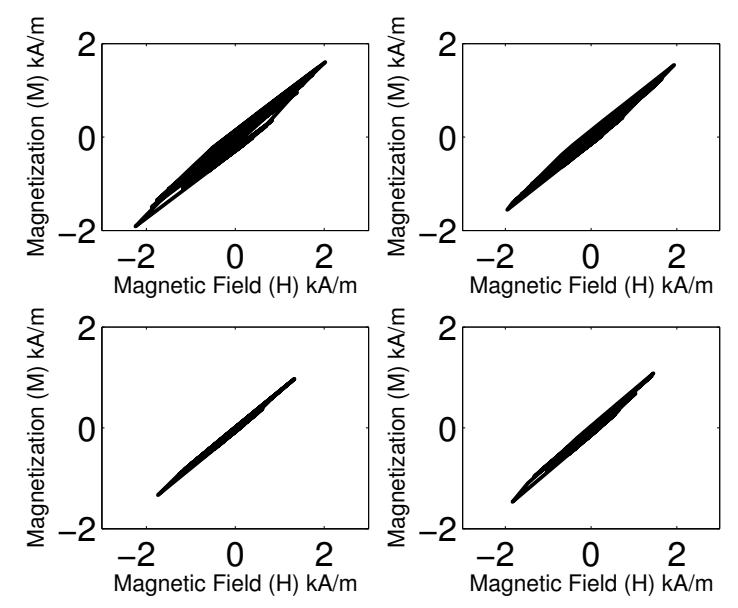

(b)

Figure 6: Performance of the linear feedback control law in the presence of a periodic external force of $25 \mathrm{~N}$. The resulting plate displacement for the uncontrolled (-) and controlled response (-) are shown in (a). The relationship between magnetic field and magnetization is shown in (b) for each actuator pair.

$$
\begin{aligned}
& F(t, z)=\left[\begin{array}{l}
A y(t)+[B(u)](t)+G(t) \\
-A^{T} \lambda(t)-Q y(t)
\end{array}\right] \\
& y\left(t_{0}\right)=y_{0} \quad, \quad \lambda\left(t_{f}\right)=\Pi_{f} y\left(t_{f}\right) .
\end{aligned}
$$

The system of equations given by (38) are solved using a finite difference discretization of the time interval $\left[t_{0}, t_{f}\right]$ with a uniform mesh having stepsize $\Delta t$ and points $t_{0}, t_{1}, \cdots, t_{N}=t_{f}$. The approximate values of $z$ at these times are denoted by $z_{0}, \cdots, z_{N}$. A central difference approximation of the temporal derivative yields the system

$$
\begin{aligned}
& \frac{1}{\Delta t}\left[z_{j+1}-z_{j}\right]=\frac{1}{2}\left[F\left(t_{j}, z_{j}\right)+F\left(t_{j+1}, z_{j+1}\right)\right] \\
& E_{0} z_{0}=\left[y_{0}, 0\right]^{T} \\
& E_{f} z_{N}=[0,0]^{T}
\end{aligned}
$$

for $j=0, \cdots, N-1$. The matrices $E_{0}$ and $E_{f}$ represent the boundary conditions at the initial and final times which are given by the relations 


$$
E_{0}=\left[\begin{array}{ll}
I & 0 \\
0 & 0
\end{array}\right] \quad, \quad E_{f}=\left[\begin{array}{cc}
0 & 0 \\
-\Pi_{f} & I
\end{array}\right] .
$$

Here $I$ denotes a $2 N_{w} \times 2 N_{w}$ identity matrix where $N_{w}$ denotes the number of basis functions employed in the spatial approximation of the state variables given in Section 3.2. The boundary conditions are written in this form to ensure the initial plate displacement and velocity are fixed according to the prescribed initial condition in (39), while the solution to $\lambda\left(t_{f}\right)$ is related to the unknown plate displacement and velocity at $t_{f}$ which must be determined according to the optimality system.

The determination of a solution vector $z_{h}=\left[z_{0}, \cdots, z_{N}\right]$ in (40) can be expressed as the problem of finding $z_{h}$ which solves

$$
\mathcal{F}\left(z_{h}\right)=0
$$

where the system of equations given in (42) is represented by $\mathcal{F}\left(z_{h}\right) \in \mathbb{R}^{4(N+1) N_{w}}$,

$$
\mathcal{F}\left(z_{h}\right)=\left[\begin{array}{c}
F_{0} \\
F_{1} \\
\vdots \\
F_{j} \\
\vdots \\
F_{N-1} \\
b\left(z_{0}, z_{N}\right)
\end{array}\right] \quad, \quad F_{j} \equiv \frac{1}{\Delta t}\left[z_{j+1}-z_{j}\right]-\frac{1}{2}\left[F\left(t_{j}, z_{j}\right)+F\left(t_{j+1}, z_{j+1}\right)\right]
$$

A quasi-Newton iteration of the form $z_{h}^{k+1}=z_{h}^{k}+\xi_{h}^{k}$, where $\xi_{h}^{k}$ solves

$$
\mathcal{F}^{\prime}\left(z_{h}^{k}\right) \xi_{h}^{k}=-\mathcal{F}\left(z_{h}^{k}\right)
$$

is used to determine the solution to the nonlinear system (42). The Jacobian $\mathcal{F}^{\prime}\left(z_{h}^{k}\right)$ has the structure

$$
\mathcal{F}^{\prime}\left(z_{h}\right)=\left[\begin{array}{cccccc}
S_{0} & R_{0} & & & & \\
& S_{1} & R_{1} & & & \\
& & \ddots & \ddots & & \\
& & & & S_{N-1} & R_{N-1} \\
& & & & & E_{f}
\end{array}\right]
$$


where

$$
\begin{aligned}
S_{i} & =-\frac{1}{\Delta t} I-\frac{1}{2} \mathcal{A}\left(t_{i}\right) \\
R_{i} & =\frac{1}{\Delta t} I-\frac{1}{2} \mathcal{A}\left(t_{i+1}\right) .
\end{aligned}
$$

The matrix $\mathcal{A}\left(t_{i}\right)$ is the linearization

$$
\mathcal{A}\left(t_{i}\right)=\frac{\partial F}{\partial z}\left(t_{i}, z_{i}\right)
$$

which yields the representation

$$
S_{i}=-\frac{1}{\Delta t}\left[\begin{array}{cc}
I & 0 \\
0 & I
\end{array}\right]-\frac{1}{2}\left[\begin{array}{cc}
A & \frac{\partial}{\partial \lambda} B\left[u_{i}^{*}\right] \\
-Q & -A^{T}
\end{array}\right]
$$

for $S_{i}$. The representation for $R_{i}$ is similar.

Direct solution of (44) is infeasible due to the large number of basis functions and time increments required to resolve the solution over a reasonable time interval. The structure of the Jacobian can be employed to reduce both memory and computational requirements to the level of solving $4 N_{w} \times 4 N_{w}$ systems. Following [39], this is accomplished by expressing the Jacobian in terms of an analytic $L U$ decomposition

$$
\mathcal{F}^{\prime}\left(z_{h}^{k}\right)=L U
$$

where

$$
L=\left[\begin{array}{ccccc}
S_{0} & & & & \\
& S_{1} & & & \\
& & & & \\
& & \ddots & & 0 \\
& & & S_{N-1} & \\
E_{0} & -E_{0}\left(S_{0}^{-1} R_{0}\right) & \cdots & E_{0} \prod_{i=0}^{N-2}(-1)^{i}\left(S_{i}^{-1} R_{i}\right) & E_{f}+E_{0} \prod_{i=0}^{N-1}(-1)^{i}\left(S_{i}^{-1} R_{i}\right)
\end{array}\right]
$$




$$
U=\left[\begin{array}{ccccc}
I & S_{0}^{-1} R_{0} & & & \\
& I & S_{1}^{-1} R_{1} & & \\
& & \ddots & \ddots & \\
& & & I & S_{N-1}^{-1} R_{N-1} \\
& & & & I
\end{array}\right] .
$$

The direct solution of the lower triangular system $L \zeta_{h}^{k}=-\mathcal{F}\left(z_{h}^{k}\right)$ is first obtained followed by direct solution of the upper triangular system $U \xi_{h}^{k}=\zeta_{h}^{k}$. This leads to the solution of the system (44).

Remark 1: The nonlinear optimal control problem includes certain approximations to improve numerical efficiency. For example, the term $\frac{\partial}{\partial \lambda} B\left[u^{*}\right]$ in $S_{i}$ and $R_{i}$ is not trivial and is assumed to be linear in the present model. This leads to a suboptimal nonlinear controller where $S_{i}=S$ and $R_{i}=R$ for all time steps. Furthermore, the condition number on the matrices $S$ and $R$ is partially dependent on the time step used in the simulations. In the simulations presented here, the time step $\Delta t=0.01 \mathrm{~s}$ was employed. Simulations using smaller time steps resulted in practically the same dynamic response until $S$ and $R$ become ill-conditioned which introduced a numerical instability. This occurred when $\Delta t<0.005 \mathrm{~s}$.

The term $B_{u}^{T}\left(u^{*}\right)$ in (26) contains nonlinearites and hysteresis domain switching which occurs at moderate to high field levels. This value can be computed by numerically by differientiating the constitutive response during the control simulation, although numerical errors may introduce instabilities. Because the control input is considered for biased minor hysteresis loops, potential numerical instabilities are mitigated by assuming the tangential magnetic susceptibility is equal to the linear coefficient $\bar{\chi}_{M}$. Unmodeled dynamics resulting from both the approximation in the Jacobian and the tangential magnetic susceptibility are addressed through perturbation feedback control presented in Section 4.3.

In Section 3, a reduction in the number of control inputs was implemented in the control design by assuming diametrically opposed magnetic fields on each top and bottom actuator pair resulted in diametrically opposed magnetization. Although this simplifies the control design, nonlinear $H-M$ behavior may give rise to differences in magnetization under moderate to high magnetic field inputs. In the present model, the unmodeled dynamics are addressed through perturbation feedback, although the control design could also be modified to include the additional degree of freedom on each actuator couple.

Remark 2: The analytic $L U$ decomposition previously developed by Smith [39] allows good spatial and time resolution of the plate dynamics. When considering the time step $\Delta t=0.01 \mathrm{~s}$ over the time interval 
$[0.45,2.50] \mathrm{s}$ and spatial approximation with $4 N_{w}=100$, approximately 30,000 unknown coefficients must be determined. The $L U$ decomposition method reduces the matrix system to solving 100 unknowns for each time step. Numerical stabilities were also checked for larger system sizes. The time step was decreased to $0.005 \mathrm{~s}$ and the number of basis functions increased to $8\left(4 N_{w}=324\right)$. This resulted in determining over 130,000 coefficients which was possible by solving the 324 coefficients for each time step separately. A stable solution was obtained that was practically identical to the one where $4 N_{w}=100$ and $\Delta t=0.01 \mathrm{~s}$ over the same time interval.

\subsubsection{Numerical Example - Truncated External Force}

Enhancement of active vibration damping is significant when the nonlinear control method is employed. In Figure 7, the same disturbance load (37) used in the linear model is applied to the Terfenol plate structure. When $t_{0}=0.45 \mathrm{~s}$, the disturbance load is again set to zero and control is instantaneously applied. By increasing the penalty on the states through $Q$ and relaxing the penalty on $u$ through $R$ as given in Table 2 , the control input reaches moderate to high drive levels. Although this creates nonlinearity and hysteresis in the Terfenol transducers similar to that shown in Figure 5(b), the nonlinear control method effectively compensates for this behavior. The plate vibration at the point $\left[\ell_{x}, \ell_{y}\right]$ is eliminated in less than half the time relative to the linear simulation in Figure 4.

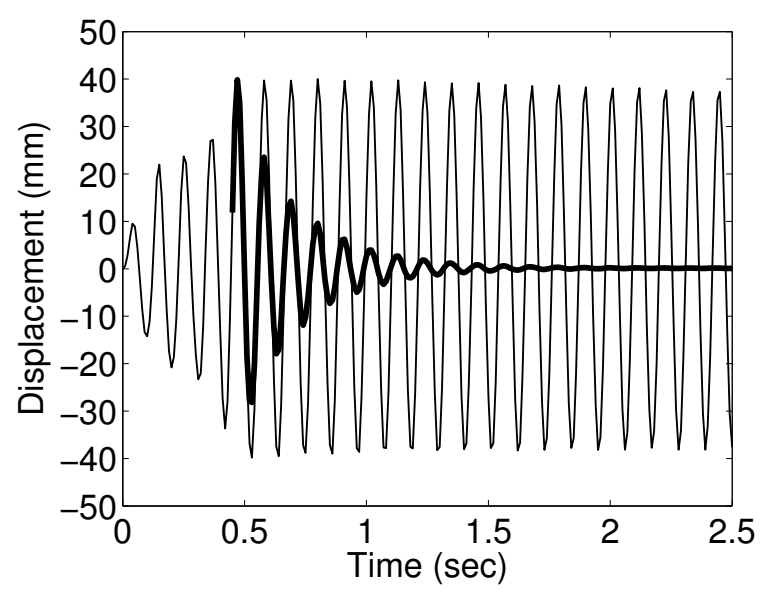

(a)
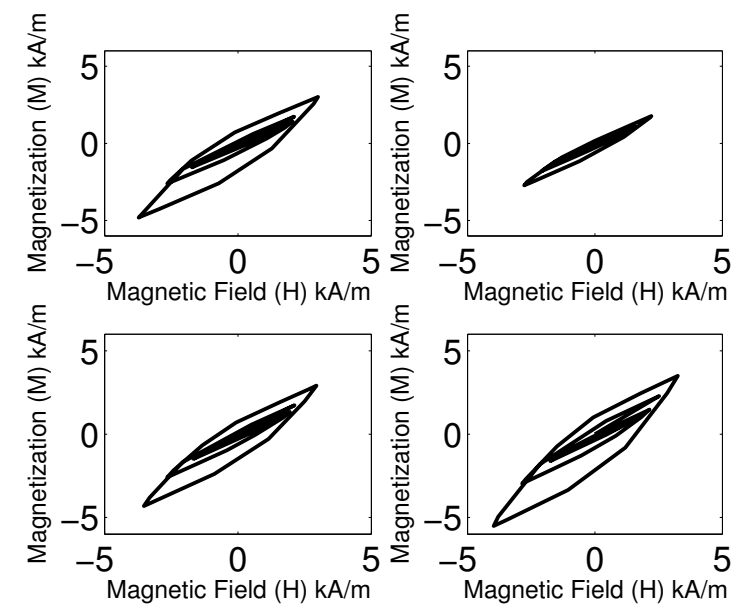

(b)

Figure 7: Nonlinear feedback control law for the open loop control. (a) Plate displacement for the uncontrolled (—) and controlled response (-). (b) Relationship between magnetization and magnetic field. 


\subsubsection{Numerical Example - Periodic External Disturbances and Operating Uncertainties}

The effect of disturbances on the plate is investigated using the nonlinear control method by applying a nonzero $G(t)$ in the optimality system given by (39). A disturbance load of $25 \mathrm{~N}$ is again applied uniformly to the plate at the previously noted frequencies. Figure 8 illustrates significantly improved vibration attenuation when the nonlinear method is employed. Further details regarding the level of vibration attenuation attainable is discussed in Remark 3 of Section 4.4.1.

The previous simulations have focused on nonlinear open loop control design which raises the question regarding robustness to operating uncertainty. To investigate this issue, the open loop control trajectory is applied $0.05 \mathrm{~s}$ late. In addition, the multiple frequencies of excitation computed in the open loop case in (37) are perturbed in the frequency domain by $+4 \mathrm{~Hz}$. In the simulation, the plate is initially excited at the unperturbed periodic force and then the frequency perturbation is applied. Continuity in the perturbed and unperturbed disturbance forces is ensured at the onset of turning on the control signal through the disturbance force

$$
g(t, x, y)= \begin{cases}25 \sum_{i=1}^{5}\left[\sin \left(2 \pi f_{i}^{e} t\right)\right], & t \leq .45 \\ 25 \sum_{i=1}^{5}\left[\sin \left(2 \pi f_{i}^{a} t-1.8 \pi\right)\right], & .45<t \leq 2.5\end{cases}
$$

where $f_{i}^{e}$ are the expected frequencies given in Section 4.1.1 and $f_{i}^{a}$ are the 'actual' frequencies shifted by $+4 \mathrm{~Hz}$. The factor $1.8 \pi$ ensures continuity of $g$.

Figure 9 illustrates complete degradation in control authority when operating uncertainties are present. In Figure 9(a) the effect of turning on the control signal $0.05 \mathrm{~s}$ is simulated. It is illustrated that complete loss of control authority occurs when open loop control is applied. In Figure 9(b), the additional effect of perturbations in the assumed force excitation is simulated. In this case, the control is again turned on $0.05 \mathrm{~s}$ late and disturbance loads from (46) are applied. Complete loss of control authority again occurs as illustrated in Figure 9(b). 


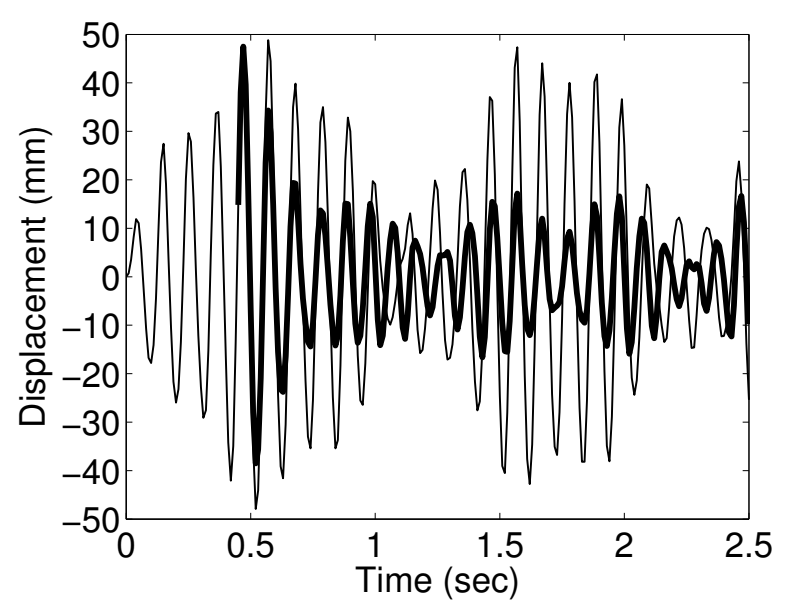

(a)
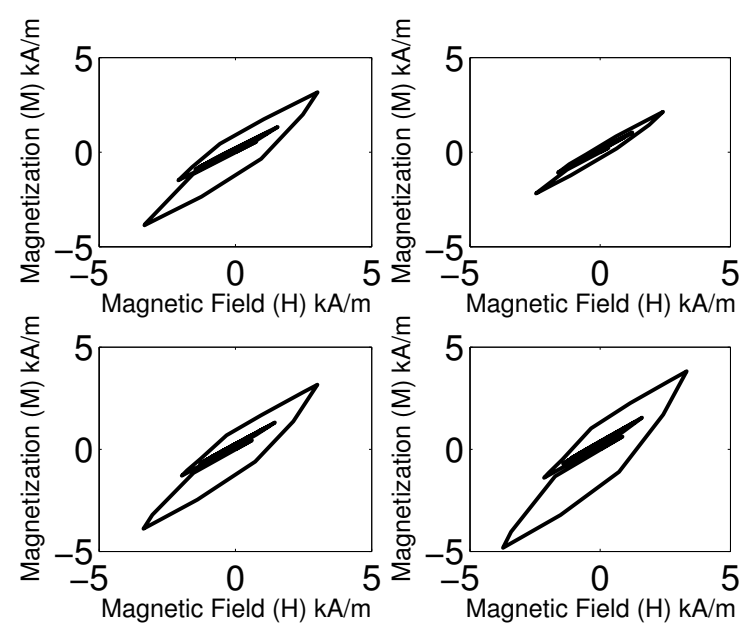

(b)

Figure 8: Performance of the nonlinear control law when disturbance loads are present. (a) Plate displacement for the uncontrolled (-) and controlled response (-). (b) Relationship between magnetization and magnetic field.

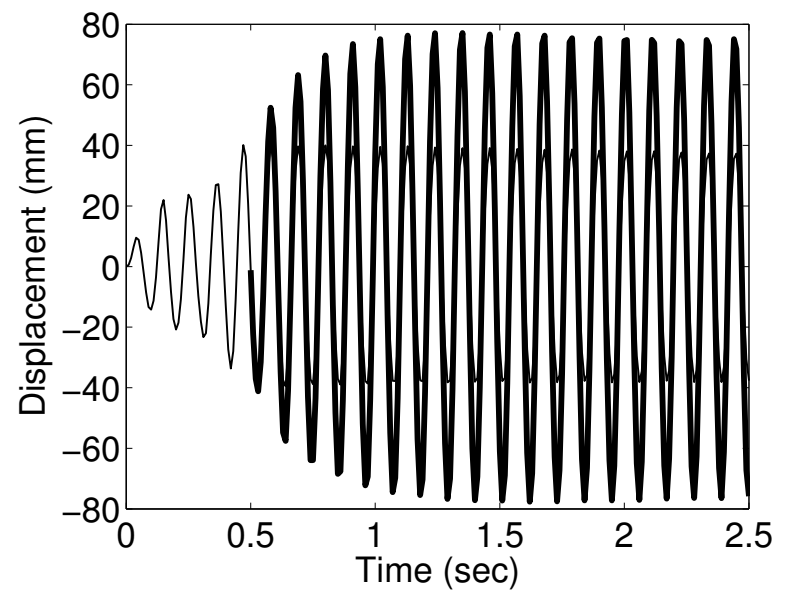

(a)

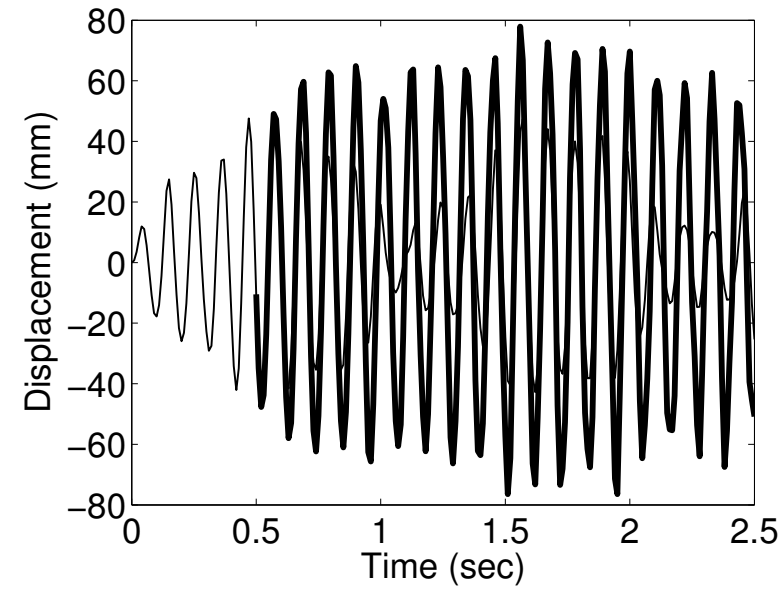

(b)

Figure 9: (a) Performance of the nonlinear control law when the control is turned on 0.05 sec late. (b) The additional effect of perturbed periodic disturbance loads. The operating uncertainty causes a complete loss of control authority. 


\subsection{Perturbation Control}

Open loop control suffers from a lack of robustness to operating uncertainties and unmodeled dynamics. It has been shown that robustness to various types of uncertainties can be significantly improved by considering perturbation control techniques [5, 27]. The feedback control input is determined from a linearization of the system about the optimal control pair $\left(u^{*}(t), y^{*}(t)\right)$. Feedback control $\delta u^{*}(t)$ is designed to attenuate perturbations in the system that may originate from external forces or initial conditions described in the previous section. Since the theory is based on a linearized system with quadratic constraints, LQR theory can be utilized. This provides an efficient algorithm for implementing the control design in real-time by first computing the open loop control offline and using online feedback of $\delta u^{*}(t)$.

The modified cost functional given by (22) and constraint given by (20) are used to determine the perturbation feedback control. Since the optimal control pair $\left(u^{*}(t), y^{*}(t)\right)$ minimizes the first order variation in $\bar{J}$, the second-order terms are expanded to determine $\left(\delta u^{*}(t), \delta y^{*}(t)\right)$. The second-order variation in $\bar{J}$ and the first-order variation in the constraint are given by,

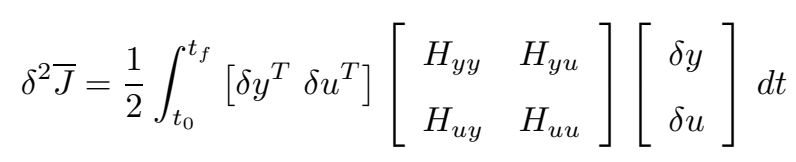

and

$$
\begin{aligned}
& \delta \dot{y}(t)=A \delta y(t)+B \delta u(t)+\delta G(t) \\
& \delta y(0)=\hat{y}_{0}
\end{aligned}
$$

where $\delta u$ and $\delta y$ are first-order variations about $u^{*}$ and $y^{*}$. The optimal perturbation control $\delta u^{*}$ is that which minimizes (47) subject to (48).

For the Hamiltonian given by (23), the second variation $\delta^{2} \bar{J}$ is,

$$
\delta^{2} \bar{J}=\frac{1}{2} \int_{t_{0}}^{t_{f}}\left\{\delta y^{T} Q \delta y+\delta u^{T} R \delta u\right\} d t
$$

so that the LQR theory outlined in Section 4.2 can be directly employed to obtain $\delta u^{*}(t)$ and $\delta y^{*}(t)$. The overall control for the system is then taken to be $u^{*}(t)+\delta u^{*}(t)$ with the optimal state given by $y^{*}(t)+\delta y^{*}(t)$.

\subsubsection{Numerical Example}

The robustness of the perturbation control method is compared to the previous example where a perturbed initial value and disturbance load are introduced. As illustrated in Figure 9, perturbation in initial conditions and external disturbances is sufficient to destroy the authority of the open loop nonlinear control. The two 
previous cases are again considered. First perturbations in only the initial value are considered and then the additional effects of perturbations in the expected periodic disturbance load are evaluated.

When perturbations in the disturbance load are absent, the control law is given by,

$$
\delta u^{*}(t)=-R^{-1} B^{T} \Pi \delta y(t)
$$

where $\Pi$ is the solution of the algebraic Ricatti equation for the perturbed system.

The perturbation in disturbance loads is represented by the relation

$$
\delta g(t, x, y)=25 \sum_{i=1}^{5}\left[\sin \left(2 \pi f_{i}^{a} t-1.8 \pi\right)-\sin \left(2 \pi f_{i}^{e} t\right)\right]
$$

$.45<t \leq 2.5$ where the 'actual' frequencies $f_{i}^{a}$ have again been shifted by $+4 \mathrm{~Hz}$ relative to the expected frequencies $f_{i}^{e}$.

The control law

$$
\delta u^{*}(t)=-R^{-1} B^{T}[\Pi \delta y(t)-\delta r(t)]
$$

compensates for the disturbance loads where $\delta r(t)$ is governed by the following auxiliary equation,

$$
\begin{aligned}
& \delta \dot{r}(t)=-\left[A-B R^{-1} B^{T} \Pi\right]^{T} r(t)+\Pi \delta G(t) \\
& \delta r(0)=\delta r(\tau)
\end{aligned}
$$

The resulting vibration control is illustrated in Figure 10. In comparison to Figure 9(a), the effect of turning on the control input $0.05 \mathrm{~s}$ late is effectively compensated using perturbation feedback as illustrated in Figure 10(a). In Figure 10(b), the additional effect of perturbations in the disturbance load is simulated. The perturbation control input computed using (52) is shown to effectively compensate for turning on the control late as well as perturbation in the disturbance loads. This demonstrates significant enhancement in control authority in comparison to Figure 9(b). It is noted that through the use of the perturbation feedback control, vibration attenuation comparable to that in the unperturbed system in Figures 7 and 8 is obtained.

Remark 3: The open loop nonlinear control and perturbation feedback simulations given in Figures 8 and 10(b) do not completely eliminate plate vibration. This is not a deficiency in the control design, but a physical limitation of the Terfenol actuators. The current location and number of actuators have not been optimized. Improvements can be made by determining the appropriate location and number of actuators for a given application. 


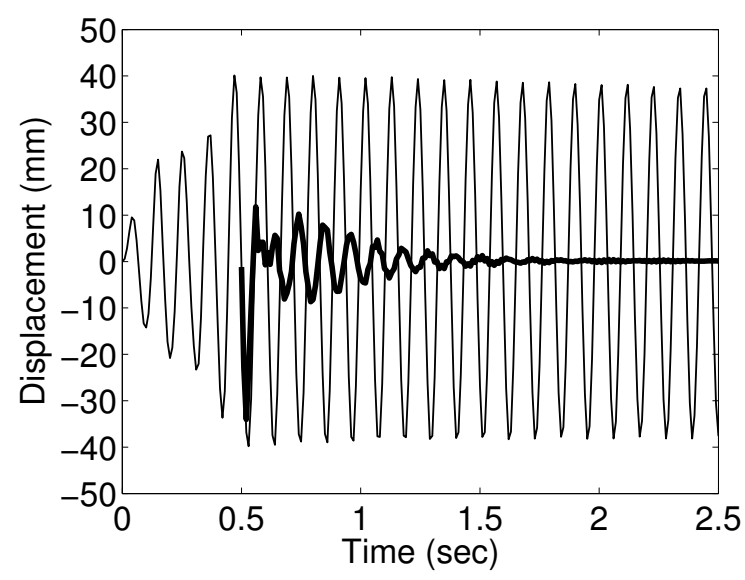

(a)

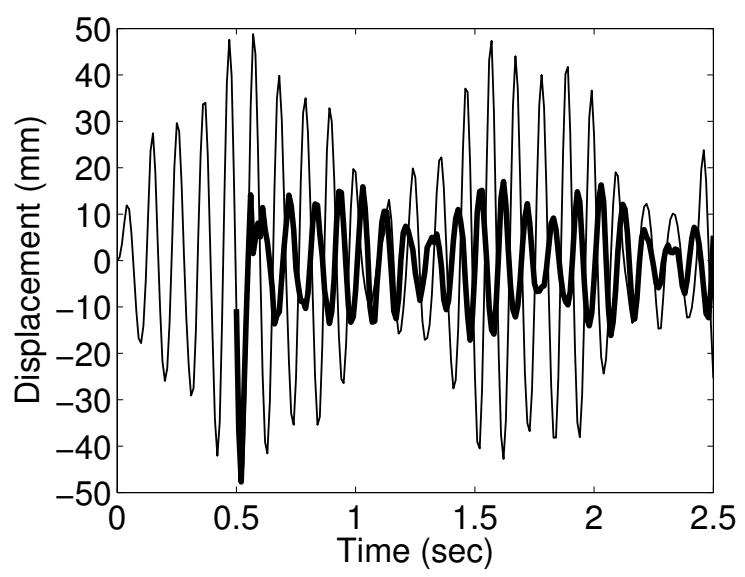

(b)

Figure 10: Performance of the perturbation feedback control law when operating uncertainties are present. (a) The open loop control is turned on $0.05 \mathrm{sec}$ late and zero disturbance loads are present. (b) The perturbation in expected disturbance loads given by (51) is included.

\section{Concluding Remarks}

A model-based nonlinear optimal control method was developed to actively attenuate plate vibration using magnetostrictive materials. While linear control methods provide marginal vibration attenuation when the input field is limited to the linear regime, performance improvements can be obtained by driving the transducers at higher field levels. While moderate to high field inputs provide the potential for improved vibration control, the resulting nonlinear and hysteretic constitutive behavior cannot be effectively compensated using linear control methods. This constitutive behavior was directly incorporated into the optimal control design which significantly improved vibration control. Moreover, robustness to operating uncertainties was obtained by feedback of perturbations around the optimal trajectory, thus providing a hybrid optimal control strategy for real-time applications.

Whereas the nonlinear control design for vibration attenuation is developed in the context of magnetic transducers, the unified nature of the framework permits direct extension of the models and model-based control methods to ferroelectric and ferroelastic compounds $[40,43,44]$. The extension of the control theory to specific PZT and SMA applications and experimental implementation of the control designs for a range of ferrioc transducers operating in hysteretic and nonlinear regimes is under present investigation.

A complementary control problem is that of tracking a reference trajectory. In the context of smart material 
actuators, such tracking problems include high accuracy and high frequency machining of out-of-round ingots using Terfenol-D transducers as well as nanoscale positioning of PZT stages in an atomic force microscope (AFM). Whereas the form of complementary 'tracking equations' is analogous to the disturbance differential equations employed here, the implementation of the tracking problems differs fundamentally in the perturbation control formulation. The development of a complementary nonlinear optimal control design for high accuracy, high frequency tracking using a Terfenol-D transducer is addressed in [30].

\section{Acknowledgements}

The authors gratefully acknowledge support by the Air Force Office of Scientific Research through the grant AFOSR-FA9550-04-1-0203.

\section{Appendix}

We summarize here the moment-displacement relations used in formulating the thin plate structure; details can be found in [40]. The moment components are given in terms of the stiffness and damping relations,

$$
\begin{gathered}
\mathcal{M}_{x}^{i n t}=-\frac{E_{p} h^{3}}{12\left(1-\nu_{p}^{2}\right)}\left(\frac{\partial^{2} w}{\partial x^{2}}+\nu_{p} \frac{\partial^{2} w}{\partial y^{2}}\right)-\frac{c_{p} h^{3}}{12\left(1-\nu_{p}^{2}\right)}\left(\frac{\partial^{2} w}{\partial x^{2} \partial t}+\nu_{p} \frac{\partial^{3} w}{\partial y^{2} \partial t}\right) \\
\mathcal{M}_{y}^{i n t}=-\frac{E_{p} h^{3}}{12\left(1-\nu_{p}^{2}\right)}\left(\frac{\partial^{2} w}{\partial y^{2}}+\nu_{p} \frac{\partial^{2} w}{\partial x^{2}}\right)-\frac{c_{p} h^{3}}{12\left(1-\nu_{p}^{2}\right)}\left(\frac{\partial^{3} w}{\partial y^{2} \partial t}+\nu_{p} \frac{\partial^{3} w}{\partial x^{2} \partial t}\right) \\
\mathcal{M}_{x y}^{i n t}=-\frac{E_{p} h^{3}}{24\left(1+\nu_{p}\right)}\left(\frac{\partial^{2} w}{\partial x \partial y}\right)-\frac{c_{p} h^{3}}{24\left(1+\nu_{p}\right)}\left(\frac{\partial^{3} w}{\partial x \partial y \partial t}\right) .
\end{gathered}
$$

These relations are substituted into the weak form of the model given by (15) to obtain the governing equations in terms of the plate displacement $w$ and the test functions $\Phi$. The approximate solution to this equation is obtained by substituting the plate displacements given in (17) into (15). This gives rise to the finite dimensional formulation in (18). The resulting mass, damping, and stiffness matrices in (18) are given by

$$
\begin{gathered}
{[\mathbb{M}]_{j k}=\int_{\Omega} \rho_{p} \Phi_{j} \Phi_{k} d \omega} \\
{[\mathbb{K}]_{j k}=\left[\mathbb{K}_{1}\right]_{j k}+\left[\mathbb{K}_{2}\right]_{j k}+\left[\mathbb{K}_{3}\right]_{j k}+\left[\mathbb{K}_{4}\right]_{j k}+\left[\mathbb{K}_{5}\right]_{j k}}
\end{gathered}
$$

where 


$$
\begin{aligned}
& {\left[\mathbb{K}_{1}\right]_{j k}=\int_{\Omega}\left(\frac{E_{p} h^{3}}{12\left(1-\nu_{p}^{2}\right)}\right) \frac{\partial^{2} \Phi_{j}}{\partial x^{2}} \frac{\partial^{2} \Phi_{k}}{\partial x^{2}} d \omega} \\
& {\left[\mathbb{K}_{2}\right]_{j k}=\int_{\Omega}\left(\frac{\nu_{p} E_{p} h^{3}}{12\left(1-\nu_{p}^{2}\right)}\right) \frac{\partial^{2} \Phi_{j}}{\partial x^{2}} \frac{\partial^{2} \Phi_{k}}{\partial y^{2}} d \omega} \\
& {\left[\mathbb{K}_{3}\right]_{j k}=\int_{\Omega}\left(\frac{E_{p} h^{3}}{12\left(1-\nu_{p}^{2}\right)}\right) \frac{\partial^{2} \Phi_{j}}{\partial y^{2}} \frac{\partial^{2} \Phi_{k}}{\partial y^{2}} d \omega} \\
& {\left[\mathbb{K}_{4}\right]_{j k}=\int_{\Omega}\left(\frac{\nu_{p} E_{p} h^{3}}{12\left(1-\nu_{p}^{2}\right)}\right) \frac{\partial^{2} \Phi_{j}}{\partial y^{2}} \frac{\partial^{2} \Phi_{k}}{\partial x^{2}} d \omega} \\
& {\left[\mathbb{K}_{5}\right]_{j k}=\int_{\Omega}\left(\frac{E_{p} h^{3}}{12\left(1+\nu_{p}\right)}\right) \frac{\partial^{2} \Phi_{j}}{\partial x \partial y} \frac{\partial^{2} \Phi_{k}}{\partial x \partial y} d \omega}
\end{aligned}
$$

The damping matrix $\mathbb{C}$ is constructed in a manner similar to the stiffness matrix $\mathbb{K}$. The disturbance load is defined by

$$
[\mathbf{g}]_{j}=\int_{\Omega} g \Phi_{j} d \omega
$$

where $g$ is the magnitude of the disturbance load and the input matrix is

$$
[\mathbf{b}]_{j i}=\int_{\Omega}\left(\chi_{x(i)}(x, y) \frac{\partial^{2} \Phi_{j}}{\partial x^{2}}+\chi_{y(i)}(x, y) \frac{\partial^{2} \Phi_{j}}{\partial y^{2}}\right) d \omega .
$$

where the characteristic functions are defined in (10) and (11). This ensures consistency with the applied moment components in the $x$ and $y$ directions.

\section{References}

[1] Banks, H.T., S.C. Beeler, G.M. Kepler, and H.T. Tran, 2002. "Reduced order modeling and control of thin film growth in a HPCVD reactor," 62(4): 1251-1280.

[2] Bittanti, S., A. Locatelli, and C. Maffezzoni, 1972. "Periodic optimization under small perturbations," Periodic Optimization, Vol. II, A. Marzollo, Ed., Udine, Springer-Verlag, New York, 183-231. 
[3] Bryant, M.D., B. Fernández, N. Wang, V.V. Murty, V.Vadlamani and T.S. West, 1992. "Active vibration control in structures using magnetostrictive Terfenol with feedback and/or neural network controllers,", Proceedings of the Conference on Recent Advances in Adaptive and Sensory Materials and their Applications, Technomic Publishing, Blacksburg, VA, April 27-29, 465-479. 5: 431-436.

[4] Bryant, M.D. and N. Wang, 1994. "Audio range controllability of linear motion Terfenol actuators," J. Intell. Mater. Syst. Struct., 5: 431-436.

[5] Bryson, A.E. and Y.-C. Ho, 1969. Applied Optimal Control, Blasidell Publishing Company, Waltham, MA.

[6] Butler, J.L. "Application manual for the design of ETREMA Terfenol-D magnetostrictive transducers," EDGE Technologies, Inc. Ames, IA.

[7] Choi, S.B., S.S., Han, and Y.S. Lee, 2005. "Fine motion control of a moving stage using a piezoactuator associated with a displacement amplifier," Smart. Mater. Struct., 14: 222-230.

[8] Calkins, F.T., R.C. Smith, A.B. Flatau, 2000. "An energy-based hysteresis model for magnetostrictive transducers," ICASE Report 97-60, IEEE T. Magn., 34(2): 429-439.

[9] Clark, A.E. 1980. "Magnetostrictive rare earth-Fe2 compounds," Chapter 7 in Ferromagnetic Materials, Vol. 1, E.P.Wohlfarth, editor, North-Holland Publishing Company, Amsterdam, 531-589.

[10] Dapino, M.J. 2002. "Magnetostrictive materials," Encyclopedia of Smart Materials, M. Schwartz, Ed., John Wiley and Sons, New York: 600-620.

[11] Dapino, M.J. 2004. "On magnetostrictive materials and their use in adaptive structures," Struct. Eng. Mech., 17(3-4): 303-329.

[12] Dapino, M.J., F.T. Calkins, and A.B. Flatau, 2000. "Magnetostrictive devices," Wiley encyclopedia of Electrical and Electronics Engineering, John G. Webster, Ed. John Wiley and sons, Inc., New York 12: 278-305.

[13] Dapino, M.J., R.C. Smith, and A.B. Flatau, 2000. "Structural magnetic strain model for magnetostrictive transducers," IEEE T. Magn., 36(3): 545-555.

[14] Da Prato, G., 1987. "Synthesis of optimal control for an infinite dimensional periodic problem," SIAM J. Control Optim., 25(3): 706-714.

[15] Della Torre, E., 1999. Magnetic Hysteresis, IEEE Press, New York. 
[16] Della Torre, E., L.H. Bennett, R.A. Fry, and Ó. Alejos. 2002. "Preisach-Arrhenius model for thermal after-effect," IEEE T. Magn., 38(5): 3409-3416.

[17] Everett, D.H. and W.I. Whitton, 1952 "A general approach to hysteresis," T. Faraday Soc., 48: 749-757.

[18] Flatau, A.B. and R. Kellog, 2004. "Blocked-force Characteristics of Terfenol-D Transducers," J. Intell. Mater. Syst. Struct., 15: 117-128.

[19] Galinaitis, W.S. and R.C. Rogers, 1997. "Compensation for hysteresis using bivariate Preisach models," Proc. SPIE Int. Soc. Opt. Eng., 3039: 538-547.

[20] Hall, D.L. and A.B. Flatau, 1998 "Analog feedback control for magnetostrictive transducer linearization," J. Sound Vib., 211(3): 481-494.

[21] Hiller, M.W., M.D. Bryant and J. Umegaki, 1989. "Attenuation and transformation of vibration through active control of magnetostrictive Terfenol," J. Sound. Vib., 134(3): 507-519.

[22] Hughes, D. and J.T. Wen, 1997. "Preisach modeling of piezoceramic and shape memory alloy hysteresis," Smart Mater. Struct. 6: 287-300.

[23] Jenner, A.G., R.D. Greenough, D. Allwood, and A.J. Wilkinson, 1994. "Control of Terfenol-D under load," J. Appl. Phys., 76(10): 7160-7162.

[24] Jiles, D.C., 1991. Introduction to Magnetism and Magnetic Materials, Chapman and Hall, New York.

[25] Jiles, D.C. and D.L. Atherton, 1986. "Theory of ferromagnetic hysteresis," J. Magn. Magn. Mater., 61: 48-60.

[26] Lee, E.B. and L. Markus, 1967. Foundations of Optimal Control Theory, John Wiley and Sons, New York.

[27] Lewis, F.L. and V.L. Syrmos, 1995. Optimal Control, John Wiley and Sons, New York.

[28] Massad, J.E. and R.C. Smith, 2003. "A domain wall model for hysteresis in ferroelastic materials," J. Intell. Mater. Syst. Struct., 14(7): 455-471.

[29] Nealis, J. and R.C. Smith, "Model-based robust control design for magnetostrictive transducers operating in hysteretic and nonlinear regimes," CRSC Technical Report: CRSC-TR03-25, IEEE T. Contr. Syst. T., submitted.

[30] Oates, W.S. and R.C. Smith, "Nonlinear optimal tracking control for magnetostrictive transducers," Proc. ASME IMECE Conference, Orlando, FL, submitted. 
[31] Pinch, E.R., 1993. Optimal Control and the Calculus of Variations, Oxford University Press, Oxford, 1993.

[32] Pradhan, S.C., 2005. "Vibration suppression of FGM shells using embedded magnetostrictive layers," Int. J. Solids Struct., 42: 2465-2488.

[33] Pratt, J. and A.B. Flatau, 1995. "Development and analysis of a self-sensing magnetostrictive actuator design," J. Intell. Mater. Syst. Struct., 6(5): 639-648.

[34] Pratt, J. and A.H. Nayfey, 1997. "Boring-bar chatter control using a two-axes active vibration absorber scheme," Proceedings of Noise-Con 97, The Pennsylvania State University, University Park, PA, June 15-17, 313-324.

[35] Preisach, F., 1935. "Uber die magnetische nachwirkung," Zeitschrift fur Physik, 94: 277-302.

[36] Restorff, J.B., 1994. "Magnetostrictive materials and devices," Encyclopedia of Applied Physics, 9: 229-244.

[37] Robert, G., D. Damjanovic and N. Setter, 2001. "Preisach modeling of piezoelectric nonlinearity in ferroelectric ceramics," J. Appl. Phys., 89(9): 5067-5074.

[38] Seelecke, S. and I. Müller, 2004. "Shape memory alloy actuators in smart structures — modeling and simulation," ASME Appl. Mech. Rev., 57(1): 23-46.

[39] Smith, R.C., 1998. "A nonlinear optimal control method for magnetostrictive actuators," J. Intell. Mater. Syst. Struct., 9(6): 468-486.

[40] Smith, R.C., 2005. Smart Material Systems: Model Development, SIAM, Philadelphia, PA.

[41] Smith, R.C., M.J. Dapino and S. Seelecke, "A free energy model for hysteresis in magnetostrictive transducers," J. Appl. Phys., 93(1): 458-466.

[42] Smith, R.C. and C.L. Hom, 1999. "Domain wall theory for ferroelectric hysteresis," J. Intell. Mater. Syst. Struct., 10(3): 195-213.

[43] Smith, R.C., S. Seelecke, M.J. Dapino, and Z. Ounaies, 2003. "A unified model for hysteresis in ferroic materials," Proc. SPIE: Smart Materials and Structures, 5049: 88-99.

[44] Smith, R.C., S. Seelecke, M.J. Dapino, and Z. Ounaies. "A unified framework for modeling hysteresis in ferroic materials," J. Mech. Phys. Solids, submitted.

[45] Smith, R.C., S. Seelecke, Z. Ounaies and J. Smith, 2003. "A free energy model for hysteresis in ferroelectric materials," J. Intell. Mater. Syst. Struct., 14(11): 719-739. 
[46] Tao, G. and P.V. Kokotović, 1996. Adaptive Control of Systems with Actuator and Sensor Nonlinearities, John Wiley and Sons, New York.

[47] Timoshenko, S. and S. Woinoshky-Krieger, 1959. Theory of Plates and Shells, 2nd ed., McGraw-Hill, Inc., New York. 